\title{
SOME RESULTS ON RATIONAL SURFACES AND FANO VARIETIES
}

\author{
Francisco Javier Gallego \\ AND \\ B. P. Purnaprajna
}

\begin{abstract}
The goal of this article is to study the equations and syzygies of embeddings of rational surfaces and certain Fano varieties. Given a rational surface $X$ and an ample and base-point-free line bundle $L$ on $X$, we give an optimal numerical criterion for $L$ to satisfy property $N_{p}$. This criterion turns out to be a characterization of property $N_{p}$ if $X$ is anticanonical. We also prove syzygy results for adjunction bundles and a Reider type theorem for higher syzygies.

For certain Fano varieties we also prove results on very ampleness and higher syzygies.
\end{abstract}

\section{INTRODUCTION}

The goal of this article is to study the equations and the syzygies of embeddings of rational surfaces and certain Fano varieties. Previously Butler, Homma, Kempf, and the authors had proved results regarding syzygies of (geometrically) ruled surfaces and surfaces of nonnegative Kodaira dimension. We will be interested in knowing under what conditions the resolution of the homogeneous coordinate ring $S / I$ of an embedded variety is "simple". More precisely we want to know under what conditions the so-called property $N_{p}$ after M. Green is satisfied. We define this property next:

Definition 0.1. Let $X$ be a projective variety. A very ample line bundle $L$ is said to satisfy property $N_{0}$ if $|L|$ embeds $X$ as a projectively normal variety. A very ample line bundle $L$ satisfies property $N_{1}$ if $L$ satisfies property $N_{0}$ and the homogeneous ideal I of the image of $X$ embedded by $|L|$ is generated by quadratic equations. Finally a very ample line bundle $L$ is said to satisfy property $N_{p}$, $p \geq 1$, if it satisfies property $N_{1}$ and the matrices in the minimal graded free resolution of $S / I$ have linear entries from the second to the $p$-th step.

Section 1 is devoted to rational surfaces. Given a rational surface $X$ and an ample and base-pointfree line bundle $L$ on $X$, we observe there is an optimal criterion for $L$ to satisfy property $N_{p}$. This criterion (cf. Theorem 1.3) depends solely on the intersection number of $L$ with $-K_{X}$, namely, $L$ satisfies property $N_{p}$ if $-K_{X} \cdot L \geq p+3$. This criterion turns out to be a characterization if $X$ is anticanonical, i.e., a rational surface with effective anticanonical class. Anticanonical surfaces have been extensively studied by several authors, among them B. Harbourne, and Theorem 1.3 improves and generalizes one of his (cf. [Hb2]).

We also study the syzygies associated to adjunction bundles and prove a Reider type theorem for higher syzygies. More precisely, given any $A_{1}, \cdots, A_{n}$ ample line bundles on an anticanonical rational surface $X$ of fixed $K_{X}^{2}$, we find a sharp bound on $n$ so that $K_{X}+A_{1}+\cdots+A_{n}$ satisfies 
property $N_{p}$. One of the easy consequences of this is Mukai's conjecture, which is in fact optimal for anticanonical surfaces with $K_{X}^{2}=1$.

Properties such as base-point-freeness and very ampleness are governed numerically, as classical results on curves and Reider's theorem on surfaces show. It is natural to ask whether the same philosophy holds for the property $N_{p}$, which are a natural generalization of base-point-freeness and very ampleness. For curves Green's theorem (cf. [G], Theorem 4.e.1), which says that a line bundle of degree greater than or equal to $2 g+p+1$ satisfies property $N_{p}$, provides an affirmative answer. For rational surfaces results in this article, namely, the already mentioned Theorem 1.3 and Theorem 1.24 , which is a Reider type result for property $N_{p}$, show us as well that property $N_{p}$ only depends on numerical criteria. In fact, if $X$ is anticanonical, Theorem 1.3 tells that property $N_{p}$ for $L$ is exclusively governed by the intersection number of $L$ with a particular curve lying on $X$, namely, an anticanonical curve. This phenomenon can be also observed in other surfaces such as elliptic ruled surfaces. Indeed, Homma and the authors gave in [Ho1], [Ho2] and [GP1] a characterization for properties $N_{0}$ and $N_{1}$ in terms of the intersection number of $L$ with a few curves lying on the elliptic surface. In this case the relevant curves are a minimal section and a fiber of the elliptic ruled surface in addition to the anticanonical curve. The authors also gave in [GP2] a criterion for property $N_{p}$ in terms of the intersection number of $L$ with the three above mentioned curves, and conjectured that those intersection numbers should also characterize property $N_{p}$ as they did characterize property $N_{0}$ and $N_{1}$. It is surprising that results of such similar spirit hold for both rational surfaces and elliptic ruled surfaces. Even though both are surfaces of Kodaira dimension $-\infty$, they differ in the fact that the Picard group of a rational surface is discrete, the same does not happen for an elliptic ruled surface. The differences can be also be seen in the methods of proof used in [GP1] and [GP2] and in this article, which are indeed very distinct.

We also prove a result (cf. Theorem 1.29) connecting property $N_{p}$, which is an extrinsic property depending on the embedding of $X$, with the "termination" of ampleness of $m K_{X}+L$. In particular we show for what $m$ the line bundle $m K_{X}+L$ stops being ample for a line bundle $L$ satisfying property $N_{p}$. The formula we obtain for such an $m$ depends on $K_{X}^{2}$ and $p$.

In Section 1 we also construct several families of examples. These examples show that all the theorems and propositions proved are sharp, and that the bounds cannot be reduced.

In Section 2 we study $n$-dimensional Fano varieties of index greater than or equal to $n-1$. Let $H$ be primitive line bundle such that $-K_{X}=i H$. First we prove Theorem 2.1, which tells exactly what property $N_{p}$ is satisfied by $H$. We prove results regarding very ampleness and on the higher syzygies of multiples of a primitive $H$ on $X$ such that $-K_{X}=i H$. We derive these syzygy results from the vanishings of certain Koszul cohomology groups on the Fano variety $X$. We reduce these vanishings to the vanishings of similar Koszul cohomology groups on lower dimensional Fano varieties. These lower dimensional are subvarieties of $X$, but since we need them to be again Fano varieties, we do not obtain them by taking subsequent hyperplane sections. We take this more indirect approach because the techniques of Section 1 do not work for these higher dimensional varieties. The reason is that the information available on the resolution of the coordinate ring of the subsequent hyperplane sections is not good enough.

Finally in Section 3 we deal with $n$-dimensional Fano varieties of index $n-3$. We first give a criterion as to when $n H$ satisfies very ampleness and property $N_{0}$ when $n \geq 2$. This criterion is actually a characterization if $n \geq 3$. Then we prove a result on the higher syzygies of multiples of 
$H$. The arguments and techniques used are similar to those used in Section 2. There is though a difference worth noting. When one is considering an $n$-dimensional Fano variety $X$ of index greater than or equal to $n-1$, one deduces the vanishing of Koszul cohomology groups on $X$ from the vanishings of similar cohomology groups on a rational surface. However, if $X$ has index $n-3$, one deduces the vanishing of Koszul cohomology groups on $X$ from the vanishings of similar cohomology groups on a Calabi-Yau threefold, and eventually, on a surface of general type. The reader might wonder about what the situation is for $n$-dimensional Fano varieties of index $n-2$. Those surfaces were studied in [GP5], where the authors obtained results which are similar to those in Sections 2 and 3 of this article.

\section{RATiOnAL SURFACES}

In this section we study property $N_{p}$ for rational surfaces. The first result is a criterion for property $N_{p}$ in terms of a very precise numerical condition, namely the intersection number of the line bundle $L$ under consideration with the anticanonical class of the surface. In order to prove this theorem we will need two preliminary results we mention now:

Observation 1.1 ([GP4], Observation 2.3). Let $X$ be a regular variety (i.e., a variety such that $\left.H^{1}\left(\mathcal{O}_{X}\right)=0\right)$. Let $E$ be a vector bundle on $X$, let $C$ be a divisor such that $L=\mathcal{O}_{X}(C)$ is globally generated line bundle and $H^{1}\left(E \otimes L^{-1}\right)=0$. If the multiplication map $H^{0}\left(E \otimes \mathcal{O}_{C}\right) \otimes H^{0}\left(L \otimes \mathcal{O}_{C}\right) \rightarrow$ $H^{0}\left(E \otimes L \otimes \mathcal{O}_{C}\right)$ surjects, then the map $H^{0}(E) \otimes H^{0}(L) \rightarrow H^{0}(E \otimes L)$ also surjects.

The second result we need is a useful lemma on the vanishing of cohomology of big and basepoint-free line bundles:

Lemma 1.2. Let $X$ be a smooth surface with $p_{g}=q=0$ and $B$ a big base-point-free line bundle on $X$ such that $-K_{X} \cdot B>0$. Then $h^{1}(B)=h^{2}(B)=0$.

Proof. Since $B$ is big and base-point-free, by Bertini, there exists smooth, irreducible $C \in|B|$. Consider

$$
0 \longrightarrow \mathcal{O}_{X} \longrightarrow B \longrightarrow B \otimes \mathcal{O}_{C} \longrightarrow 0
$$

Since $p_{g}=0, h^{2}(B)=0$. Since $-K_{X} \cdot B>0$, by adjunction $\operatorname{deg}\left(B \otimes \mathcal{O}_{C}\right)>2 g(C)-2$, and consequently, $h^{1}(B)=0$, since $q=0$.

We are now ready to state and prove the numerical criterion for property $N_{p}$. This criterion turns out to be a characterization of property $N_{p}$ if the surface is anticanonical. We remark that the case of property $N_{0}$ was observed by B. Harbourne (cf. [Hb2]).

Theorem 1.3. Let $X$ be a rational surface and let $L$ be an ample line bundle on $X$. If $L$ is basepoint-free and $-K_{X} \cdot L \geq p+3$, then $L$ satisfies property $N_{p}$. In addition, if $X$ is anticanonical and $L$ is ample, then $L$ satisfies property $N_{p}$ if and only if $-K_{X} \cdot L \geq p+3$.

Proof. First we prove the part of the result stated for general rational surfaces, and we start showing that if $-K_{X} \cdot L \geq 3, L$ satisfies property $N_{0}$. We want to show that

$$
H^{0}(r L) \otimes H^{0}(L) \stackrel{\alpha}{\longrightarrow} H^{0}((r+1) L) \text { for all } r \geq 1 \text {. }
$$

Since $L$ is ample and base-point-free, we can choose a smooth and irreducible curve $C \in|L|$. Then, by Lemma 1.2 and Observation 1.1, the surjectivity of $\alpha$ follows from the surjectivity of

$$
H^{0}\left(r L_{C}\right) \otimes H^{0}\left(L_{C}\right) \stackrel{\beta}{\rightarrow} H^{0}\left((r+1) L_{C}\right) \text { for all } r \geq 1
$$


Since $-K_{X} \cdot L \geq 3, \operatorname{deg} L_{C} \geq 2 g(C)+1$, hence by Castelnuovo's Theorem $\beta$ surjects. This proves that $L$ satisfies property $N_{0}$.

Now we prove the result for general $p$. We have just proven that if $-K_{X} \cdot L \geq p+3, L$ satisfies property $N_{0}$, i.e., $L$ is very ample and embeds $X$ as a projectively normal variety. On the other hand, by Lemma 1.2, Kodaira Vanishing Theorem and because $X$ is regular, $H^{1}(r L)=0$ for all $r \in \mathbf{Z}$. Therefore the image $Y$ of $X$ by the embedding induced by $|L|$ is arithmetically Cohen-Macaulay. Let $H$ be a general hyperplane of $\mathbf{P}^{N}$ and let $D$ be the corresponding (smooth, irreducible) divisor on $X$. Since $X$ is regular, $\left|L_{D}\right|$ embeds $D$ in $H$ and the image of this embedding is $Y \cap H$, which is projectively normal. This is the same as saying that $L_{D}$ satisfies property $N_{0}$. Moreover, since $Y$ is arithmetically Cohen-Macaulay, the minimal resolution of $Y \cap H$ has the same graded Betti as the minimal resolution of $Y$ (see also [G], Theorem 3.b.7). Since $-K_{X} \cdot L \geq p+3$ we have by adjunction that $\operatorname{deg} L_{D} \geq 2 g+p+1$, then by Green's theorem (cf. [G], Theorem 4.a.1) the homogeneous coordinate ring of $Y \cap H$ is generated by quadrics and the resolution of its homogeneous coordinate ring is linear until the $p$-th step. Since we did already prove that $L$ satisfied property $N_{0}$, we see that $L$ satisfies property $N_{p}$.

Let now $X$ be anticanonical. On an anticanonical surface a nef line bundle $L$ such that $-K_{X} \cdot L \geq 2$ is base-point-free (cf. [Hb1], Theorem III.1). Therefore we have just proven one of the implications, namely that if $-K_{X} \cdot L \geq p+3$, then $L$ satisfies property $N_{p}$. We prove now the other implication. Let $L$ be a line bundle on $X$ satisfying property $N_{p}$. In particular $L$ is very ample and embeds $X$ as a projectively normal variety. Moreover, $H^{1}(r L)=0$ for all $r \in \mathbf{Z}$. Indeed, $H^{1}\left(\mathcal{O}_{X}\right)=0$ because $X$ is rational and $H^{1}(r L)=0$ by Kodaira Vanishing Theorem if $r$ is negative. If $r$ is positive, $-K_{X} \cdot r L>0$, because $L$ is ample and $-K_{X}$ is effective. Hence $H^{1}(r L)=0$ by Lemma 1.2 . Therefore the image of the embedding of $X$ by $|L|$ is arithmetically Cohen-Macaulay. Let $C$ be a smooth curve in $|L|$. As observed before, $L$ satisfies property $N_{p}$ if and only if $L_{C}$ satisfies property $N_{p}$. Since $-K_{X}$ is effective and $C$ moves (for $L$ is very ample), then $-K_{X} \otimes \mathcal{O}_{C}$ is also effective. Hence $L_{C}=K_{C}+N$ with $N$ effective line bundle of degree $-K_{X} \cdot L$. Now if $-K_{X} \cdot L$ were less than or equal to $p+2, L_{C}$ would not satisfy property $N_{p}$. This follows from a result of Green and Lazarsfeld (cf. [GL], Theorem 2) which says in particular that on a smooth, irreducible, genus $g$ curve $C$, a line bundle of the form $K_{C}+N$ with $N$ effective of degree $p+2$ does not satisfies property $N_{p}$.

Observation 1.4. If $X$ is a rational surface which is not anticanonical and $L$ is a line bundle on $X$ which satisfies $N_{p}$, in general, $-K_{X} \cdot L$ need not be greater than or equal to $p+3$. However, there are line bundles $L$ such that $-K_{X} \cdot L=p+3$ and $L$ satisfies $N_{p}$ and not $N_{p+1}$. Hence Theorem 1.3 is sharp for non-anticanonical surfaces.

Proof. Consider a non-anticanonical rational surface $X$ and let $L$ be an ample and base-point-free line bundle so that $K_{X}+L$ is also ample, and such that the general curve in $|L|$ is not hyperelliptic. Then $-K_{X} \cdot L=p+3$ for some $p$. Let $C$ be general and therefore smooth and irreducible curve in $|L|$. We know by Theorem 1.3 that $L$ satisfies property $N_{p}$. Since $K_{X}+L$ is ample, by Kodaira vanishing and duality, $H^{1}\left(-K_{X}-L\right)=0$. Since $X$ is not anticanonical, $-K_{X} \otimes \mathcal{O}_{C}$ is non effective. Then, arguing as in the proof of Theorem 1.3 we conclude, using [GL], Theorem 2, that $L$ satisfies property $N_{p+1}$.

Now we show by means of an example the existence of $(X, L)$, where $X$ is a non-anticanonical rational surface and $L$ is an ample and free line bundle such that $L$ satisfies $N_{p}$ but not $N_{p+1}$ and $-K_{X} \cdot L=p+3$. Let $\pi: X \longrightarrow \mathbf{F}_{0}$ be the blowing up of $\mathbf{F}_{0}$ at 9 points. We choose the 9 points $p_{1}, \ldots p_{9}$ so that $X$ is not anticanonical. Let $E_{1}, \ldots, E_{9}$ be the exceptional divisors and let 
$L=\pi^{*}\left(2 C_{0}+n f\right)-E_{1}-\cdots-E_{9}$ with $n \geq 4$. Then $L$ is ample, because $L=A+\pi^{*}((n-3) f)$, where $A$ is as in Example 1.16. $L$ is also base-point-free. This can be checked using Reider's Theorem. Indeed, $L$ can be written as $K_{X}+2 A+\pi^{*}((n-4) f)$. Let $C$ be a smooth curve in $|L|$. We want to show that $-K_{X} \otimes \mathcal{O}_{C}$ is effective. We look at the long exact sequence relating the cohomology of $-K_{X}-L,-K_{X}$ and $-K_{X} \otimes \mathcal{O}_{C}$. By our choice of $p_{1}, \ldots, p_{9}, h^{0}\left(-K_{X}\right)=0$ and by Riemann-Roch $h^{1}\left(-K_{X}\right)=1$. Also by Riemann-Roch, $h^{1}\left(-K_{X}-L\right)=n-3$. Then $h^{0}\left(-K_{X} \otimes \mathcal{O}_{C}\right)=n-3 \geq 1$. Now $-K_{X} \cdot L=2 n-5$. Then according to Theorem $1.3 L$ satisfies property $N_{2 n-8}$ and by [GL], Theorem $2, L$ does not satisfy property $N_{2 n-7}$.

Remark 1.5. Theorem 1.3 is an example of the following philosophy: On a variety $X$ the failure of a line bundle $L$ to satisfy property $N_{p}$ can be traced to the existence of an extremal curve $C$ on $X$. The curve $C$ is extremal with respect to $L$ and property $N_{p}$ in the following sense: $L_{C}$ satisfies property $N_{p}$ but not property $N_{p+1}$. In the proof of Theorem 1.3 the existence of such an extremal curve, namely a smooth curve $C$ in $|L|$, plays a key role. We would like to point out the existence of another extremal curve on $X$. If there is a smooth irreducible curve $C^{\prime}$ in $\left|-K_{X}\right|$ (for instance, if $X$ is a Del Pezzo surface), then $C^{\prime}$ is a smooth elliptic curve. On an elliptic curve a line bundle satisfies property $N_{p}$ if and only if its degree is greater than or equal to $p+3$. This follows from Green's theorem (cf. [G], Theorem 4.a.1) and the self-duality of the resolution of any elliptic normal curve or alternatively by the theorem of Green and Lazarsfeld quoted above (cf. [GL], Theorem 2). Now Theorem 1.3 says precisely that $L$ satisfies property $N_{p}$ but not property $N_{p+1}$ if and only if $-K_{X} \cdot L=p+3$. This agrees with the philosophy just stated, since $-K_{X} \cdot L$ is the degree of $L_{C^{\prime}}$ and $p+3$ is the degree of those line bundles of $C^{\prime}$ satisfying property $N_{p}$ but not property $N_{p+1}$.

In the remainder of this section we will focus on anticanonical surfaces. One of our main purposes is to find uniform and optimal bounds on $n$ so that a line bundle of the form $K_{X}+A_{1}+\cdots+A_{n}$ satisfies property $N_{p}$, where each $A_{i}$ is an ample line bundle on $X$. The bounds we will obtain will be for anticanonical surfaces with a fixed value of $K_{X}^{2}$. They will therefore be finer than a uniform bound valid for any anticanonical surface. There are two ingredients we need to attack the problem. First we need to find a sharp, uniform lower bound on $n$ so that $K_{X}+A_{1}+\cdots+A_{n}$ be very ample. This will be dealt with in Proposition 1.6. Second, because of the numerical characterization of property $N_{p}$ given by Theorem 1.3 , we need to find a sharp, uniform lower bound for $-K_{X} \cdot A$ for an ample $A$. This is what we do in Proposition 1.9 and Proposition 1.10. The sharpness of Propositions 1.6, 1.9 and 1.10 is shown by the Examples 1.11 to 1.20 presented later on.

Proposition 1.6. Let $X$ be an anticanonical surface, let $A_{1}, \ldots, A_{n}$ be ample line bundles on $X$, and let $L=K_{X}+A_{1}+\cdots+A_{n}$. Then $L$ is very ample if

1) $K_{X}^{2}=9$ and $n \geq 4$.

2) $K_{X}^{2}=8$ and $n \geq 3$.

3) $3 \leq K_{X}^{2} \leq 7$ and $n \geq 2$.

4) $K_{X}^{2}=2$ and $n \geq 3$ or $n \geq 2$ unless $n=2, A_{1}=A_{2}=-K_{X}$.

5) $K_{X}^{2}=1$ and $n \geq 4$ or $n \geq 2$ unless

5a) $n=2$ and $A_{1}=A_{2}=-K_{X}$ or $A_{1}=-K_{X}$ and $A_{2}=-2 K_{X}$ (or vice versa); or

5b) $n=3$ and $A_{1}=A_{2}=A_{3}=-K_{X}$.

6) $K_{X}^{2}=0$ and $n \geq 3$.

7) $K_{X}^{2}<0$ and $n \geq 2$.

In order to prove the result we need this 
Lemma 1.7. Let $X$ be an anticanonical surface and let $A$ be an ample line bundle on $X$. Then $A^{2} \geq 2$ unless $(X, A)=\left(\mathbf{P}^{2}, \mathcal{O}_{\mathbf{P}^{2}}(1)\right)$ or $K_{X}^{2}=1$ and $A=-K_{X}$.

Proof. If $-K_{X} \cdot A \geq 2$, then by [Hb1], Theorem III.1.a $A$ is base-point-free, hence if $A^{2}=1$, then $(X, A)=\left(\mathbf{P}^{2}, \mathcal{O}_{\mathbf{P}^{2}}(1)\right)$. If $-K_{X} \cdot A=1$ and $A^{2}=1$, then $\left(K_{X}+A\right) \cdot A=0$ and hence $K_{X}+A$ is effective by Riemann-Roch. Since $A$ is ample, $A=-K_{X}$ and $K_{X}^{2}=1$.

(1.8) Proof of Proposition 1.6. First, we note that if $n \geq 4$, then $L$ is very ample by Reider's Theorem. Now, except if $(X, A)=\left(\mathbf{P}^{2}, \mathcal{O}_{\mathbf{P}^{2}}(1)\right)$ or $A=-K_{X}$ and $K_{X}^{2}=1$, an ample line bundle $A$ satisfies $A^{2} \geq 2$ by Lemma 1.7. Therefore if $n \geq 3, L$ is very ample by Reider's Theorem unless all $A_{i}=A$ and, either $(X, A)=\left(\mathbf{P}^{2}, \mathcal{O}_{\mathbf{P}^{2}}(1)\right)$, or $A=-K_{X}$ and $K_{X}^{2}=1$.

Now we prove that under the hypothesis stated in the proposition, $L$ is very ample if $n \geq 2$. First we show that the result is true if $A_{1}=A_{2}=A$ and $A^{2} \geq 3$. By Reider's Theorem the only obstacle to the very ampleness of $L$ would be the existence of an irreducible curve $E$ with $A \cdot E=1$ and $E^{2}=0$. Then we prove the following:

(1.8.1) Let $X$ be an anticanonical surface with $K_{X}^{2} \neq 0,8$ and let $A$ be an ample line bundle on $X$. Then there does not exist an irreducible curve $E$ on $X$ with $A \cdot E=1$ and $E^{2}=0$.

We show that, under the above hypothesis, $p_{a}(E)=0$ or 1 . Since $E$ is irreducible and $E^{2}=0$, $E$ is nef, hence $-K_{X} \cdot E \geq 0$ and $\left(K_{X}+E\right) \cdot E \leq 0$. We exclude both possibilities, starting with $p_{a}(E)=1$. In this case $-K_{X} \cdot A=1$, otherwise $A$ would be base-point-free by [Hb1], Theorem III.1 and this would contradict the fact that $A \cdot E=1$. Now $K_{X}+E$ is effective by Riemann-Roch. Indeed, $\left(K_{X}+E\right) \cdot E=0$ and $E$ is a curve, hence $h^{0}\left(K_{X}+E\right)=1+h^{1}\left(K_{X}+E\right) \geq 1$. On the other hand $\left(K_{X}+E\right) \cdot A=0$, hence $E=-K_{X}$ and $K_{X}^{2}=0$, which is excluded by hypothesis. Now we rule out the second possibility, namely, $p_{a}(E)=0$. In this case $E=\mathbf{P}^{1}$ and it follows from Lemma 4.1.10 in $[\mathrm{BS}]$ that $X$ is a $\mathbf{P}^{1}$-bundle, which is also excluded by hypothesis.

By Lemma 1.7 the only case left when $A_{1}=A_{2}=A$ is $A^{2}=2$. Then $-K_{X} \cdot A \geq 2$ and $A$ is base-point-free by [Hb1], Theorem III.1.a. Then $X$ is either $\mathbf{P}^{1} \times \mathbf{P}^{1}$ or a double cover of $\mathbf{P}^{2}$ ramified along a smooth quartic, for which $A=-K_{X}$ and $K_{X}^{2}=2$. Both possibilities are excluded by hypothesis.

Now we deal with the case $A_{1} \neq A_{2}$. If one $A_{i}$ is such that $A_{i}^{2} \geq 5$, by (1.8.1) and Reider's Theorem $K_{X}+A_{1}+A_{2}$ is very ample. If, say, $A_{1}^{2}=4$ and $A_{2}^{2} \geq 2$, we prove the following:

(1.8.2) Let $X$ be an anticanonical surface and let $A_{1}$ and $A_{2}$ be ample line bundles on $X$ such that $A_{1}^{2} \geq 3$. Then $A_{1} \cdot A_{2} \geq 2$.

Then we are done by (1.8.1), (1.8.2) and Reider's Theorem. Therefore we prove (1.8.2). Assume the contrary. Now for any ample bundle $A$ on $X$, and in particular for $A_{2}$, we have that $h^{2}(A)=$ $h^{0}\left(K_{X}-A\right)=0$, because any ample bundle intersects $K_{X}-A$ strictly negatively. Then by RiemannRoch $A_{2}$ is effective. On the other hand $\left(K_{X}+2 A_{1}\right) \cdot A_{2} \leq 1$. We have seen before that $K_{X}+2 A_{1}$ is very ample, therefore $\left(K_{X}+2 A_{1}\right) \cdot A_{2}=1,-K_{X} \cdot A_{2}=1$ and each member of $\left|A_{2}\right|$ is a smooth $\mathbf{P}^{1}$. But if $-K_{X} \cdot A_{2}=1$ then $\left(K_{X}+A_{2}\right) \cdot A_{2} \geq 0$ and this contradicts the fact that the sectional genus of $A_{2}$ is 0 .

Now if, say, $A_{1}^{2}=4$ and $A_{2}^{2}=1$, again we know by (1.8.2) that $A_{1} \cdot A_{2} \geq 2$, and if $A_{1} \cdot A_{2} \geq 3$ we are again done by (1.8.1) and Reider's Theorem. Thus we consider the case $A_{1}^{2}=4, A_{2}^{2}=1$ and $A_{1} \cdot A_{2}=2$. Since $n=2$ and $A_{2}^{2}=1$, by Lemma $1.7 A_{2}=-K_{X}$ and $K_{X}^{2}=1$. Then $A_{1} \cdot\left(2 K_{X}+A_{1}\right)=$ 0 . On the other hand $h^{2}\left(2 K_{X}+A_{1}\right)=h^{0}\left(-K_{X}-A_{1}\right)=0$, because $A_{1} \cdot\left(-K_{X}-A_{1}\right)=-2$. Then it follows by Riemann-Roch that $2 K_{X}+A_{1}$ is effective, so $A_{1}=-2 K_{X}$. However the possibility $K_{X}^{2}=1, A_{2}=-K_{X}$ and $A_{1}=-2 K_{X}$ is excluded by hypothesis. 
Now the only remaining cases are $A_{1}^{2}=3$ and $A_{2}^{2}=1,2,3, A_{1}^{2}=2$ and $A_{2}^{2}=2,1$ and $A_{1}^{2}=A_{2}^{2}=1$. We analyze them case by case. If $A_{1}^{2}=A_{2}^{2}=3$, then by (1.8.2) $A_{1} \cdot A_{2} \geq 2$, then by (1.8.1) and Reider's Theorem we are done. If $A_{1}^{2}=3$ and $A_{2}^{2}=2$, then $-K_{X} \cdot A_{2} \geq 2$ and as already argued $A_{2}=-K_{X}$ and $K_{X}^{2}=2$. If $A_{1}^{2}=3$ and $A_{2}^{2}=1$, then by Lemma $1.7, A_{2}=-K_{X}$ and $K_{X}^{2}=1$. But then in neither of the cases can it happen that $A_{1}^{2}=3$ and $-K_{X} \cdot A_{1}=2$. If $A_{1}^{2}=2$ and $A_{2}^{2}=2$ or 1, then either $A_{1}=A_{2}=-K_{X}$ and $K_{X}^{2}=2$, which is excluded because we are assuming $A_{1} \neq A_{2}$, or $A_{1}=-K_{X}$ and $K_{X}^{2}=2$ and $A_{2}=-K_{X}$ and $K_{X}^{2}=1$, which is absurd. Finally if $A_{1}^{2}=A_{2}^{2}=1$, then by Lemma $1.7, A_{1}=A_{2}=-K_{X}$ and $K_{X}^{2}=1$, but we are assuming $A_{1} \neq A_{2}$.

We now proceed to study lower bounds for $-K_{X} \cdot A$. One lower bound is of course 1 . This bound is sharp if $K_{X}^{2} \leq 1$, as proven by Examples 1.15, 1.18, 1.19 and 1.20 . However, if $K_{X}^{2} \geq 2$ a better bound holds: First, if $K_{X}^{2}=9$, then $X=\mathbf{P}^{2}$ and the bound is 3 . If $K_{X}^{2}=8, X$ is a Hirzebruch surface and we summarize the bounds on $-K_{X} \cdot A$ in the next proposition. Finally if $1 \leq K_{X}^{2} \leq 7$, $-K_{X} \cdot A \geq K_{X}^{2}$. This bound is sharp as shown in Examples 1.13 and 1.14. We end this study by classifying the boundary and near-boundary cases when $1 \leq K_{X}^{2} \leq 7$ and by obtaining a better bound when $(X, A)$ does not fall in one of these boundary and near-boundary cases. As explained before, we need these technical results to prove Theorem 1.23.

Proposition 1.9. Let $X$ be an anticanonical surface and let $A$ be an ample line bundle on $X$. If $K_{X}^{2}=8$, i.e., if $X$ is a (geometrically) ruled rational surface, then $-K_{X} \cdot A \geq 4$. More precisely, if $X=\mathbf{F}_{e}$, then $-K_{X} \cdot A \geq e+4$.

Proof. If $X=\mathbf{F}_{e},-K_{X}$ is linearly equivalent to $2 C_{0}+(e+2) f$, where $C_{0}$ is the minimal section of $\mathbf{F}_{e}$ and $f$ is a fiber. In any case there is a divisor in the anticanonical class having $e+4$ irreducible components counted with multiplicity. Then the intersection number of any ample line bundle $A$ with this divisor is greater than or equal to $e+4$.

Proposition 1.10. Let $X$ be an anticanonical surface and let $A$ be an ample line bundle such that $1 \leq K_{X}^{2} \leq 7$.

Then $-K_{X} \cdot A \geq K_{X}^{2}+3$ unless one of the following happens:

a) $A=-K_{X}$, in which case $-K_{X} \cdot A=K_{X}^{2}$;

b) $K_{X}^{2}=1$ and $A=-2 K_{X}$, in which case $-K_{X} \cdot A=K_{X}^{2}+1$;

c) $K_{X}^{2}=1$ and $A=-3 K_{X}$, in which case $-K_{X} \cdot A=K_{X}^{2}+2$;

d) $K_{X}^{2}=2$ and $A=-2 K_{X}$, in which case $-K_{X} \cdot A=K_{X}^{2}+2$;

e) $K_{X}+A$ is a base-point-free line bundle, $A$ is very ample and $(X, A)$ is a conic fibration under $\left|K_{X}+A\right|$, in which case $-K_{X} \cdot A \geq K_{X}^{2}+2$.

Proof. We assume throughout the proof that $A \neq-K_{X}$ and we want to see that, except for the other exceptions listed in the statement, $-K_{X} \cdot\left(K_{X}+A\right) \geq 3$. We divide the proof in two cases:

Case 1: $K_{X}^{2} \geq 2$. Assume first that $A^{2} \geq 5$. Then by Hodge Index Theorem $-K_{X} \cdot A \geq 4$; in particular, $A$ is very ample. We apply Reider's Theorem to $K_{X}+A$ to see it is base-point-free. The only obstruction to $K_{X}+A$ being base-point-free is the existence of a reduced and irreducible curve $E$ such that $A \cdot E=1$ and $E^{2}=0$. Since $A$ is very ample, $E=\mathbf{P}^{1}$. Then it follows from [BS], Lemma 4.1.10 that $X$ would be a $\mathbf{P}^{1}$-bundle, which is excluded by hypothesis. We apply now [Hb1], Lemma II.6.a. If $K_{X}+A$ is composed with a pencil, since $K_{X}+A$ is base-point-free and $A$ is ample, $\left(K_{X}+A\right)^{2}=0, h^{1}\left(K_{X}+A\right)=0$ and $-K_{X} \cdot\left(K_{X}+A\right)=2 r$, for some $r \geq 1$. If $r \geq 2$, we are done. If $r=1$, then $\left(K_{X}+A\right)^{2}=0$ and $-K_{X} \cdot\left(K_{X}+A\right)=2$. Hence $\left(K_{X}+A\right) \cdot A=2$, therefore the sectional genus of $A$ is 2 .

Then by [BS], Theorem 10.2.7.2 $(X, A)$ is a conic fibration over $\mathbf{P}^{1}$ under $\left|K_{X}+A\right|$. Note that in this case $-K_{X} \cdot\left(K_{X}+A\right)$ is an even number greater than or equal to 2 . 
If $K_{X}+A$ is not compose with a pencil then $\left(K_{X}+A\right)^{2}>0$ by [Hb1], Lemma II.6.b. We want to see under what conditions $-K_{X} \cdot\left(K_{X}+A\right) \geq 3$. Since $K_{X}^{2} \geq 2$ it follows from Hodge Index Theorem that $-K_{X} \cdot\left(K_{X}+A\right) \geq 2$. Assume then that $-K_{X} \cdot\left(K_{X}+A\right)=2$. Then $\left(K_{X}+A\right)^{2} \geq 2$ and even. Now if $\left(K_{X}+A\right)^{2} \geq 4$ or $K_{X}^{2} \geq 3$, we are done by Hodge Index Theorem. Then the only case left is $\left(K_{X}+A\right)^{2}=K_{X}^{2}=2$.

On the other hand $K_{X}+A$ is big and nef, therefore by Kawamata-Viehweg $h^{1}\left(2 K_{X}+A\right)=$ $h^{2}\left(2 K_{X}+A\right)=0$ and by Riemann-Roch $2 K_{X}+A$ is effective. Since $A$ is ample and $A \cdot\left(2 K_{X}+A\right)=0$, this implies that $A=-2 K_{X}$.

We deal now with the case $A^{2} \leq 4$. By Lemma 1.7, $A^{2} \geq 2$, and by Hodge Index Theorem $-K_{X} \cdot A \geq 2$, hence by [Hb1], Theorem III.1.a $A$ is base point free. If $A^{2}=2$, then either $X=\mathbf{P}^{1} \times \mathbf{P}^{1}$ or $K_{X}^{2}=2$ and $A=-K_{X}$. The two possibilities are excluded either by hypothesis or by assumption. Let now $A^{2}=3$ or 4 .

If $A^{2}=3$, by Hodge Index Theorem $-K_{X} \cdot A \geq 3$, hence by Theorem $1.3, A$ is very ample. Then $X$ is a Del Pezzo cubic surface in $\mathbf{P}^{3}$ and $A=-K_{X}$ or a rational normal scroll in $\mathbf{P}^{4}$. The two possibilities are excluded by hypothesis or by assumption.

Finally, if $A^{2}=4$, again by Hodge Index Theorem $-K_{X} \cdot A \geq 3$ and $A$ is very ample. However the only linearly normal smooth surfaces of degree 4 are K3 surfaces in $\mathbf{P}^{3}$, the Del Pezzo surface in $\mathbf{P}^{4}$ and the Veronese Surface and the rational normal scrolls in $\mathbf{P}^{5}$.

Case 2: $K_{X}^{2}=1$. Assume first that $A^{2} \geq 5$. Then by Hodge Index Theorem $-K_{X} \cdot A \geq 3$; in particular, $A$ is very ample. The same argument used in Case 1 proves that $K_{X}+A$ is base-pointfree. We apply again [Hb1], Lemma II.6.a. Then if $K_{X}+A$ is composed with a pencil, we get as before that either $-K_{X} \cdot\left(K_{X}+A\right) \geq 4$ or, $-K_{X} \cdot\left(K_{X}+A\right)=2$ and by [BS], Theorem 10.2.7.2 $(X, A)$ is a conic fibration over $\mathbf{P}^{1}$ under $\left|K_{X}+A\right|$.

If $K_{X}+A$ is not compose with a pencil then $\left(K_{X}+A\right)^{2}>0$ by [Hb1], Lemma II.6.b. We want to see under what conditions $-K_{X} \cdot\left(K_{X}+A\right)<3$. If $\left(K_{X}+A\right)^{2} \geq 5$, then $-K_{X} \cdot\left(K_{X}+A\right) \geq 3$ by Hodge Index Theorem. Then we have to study the cases $1 \leq\left(K_{X}+A\right)^{2} \leq 4$. Then by Hodge Index Theorem $-K_{X} \cdot\left(K_{X}+A\right) \geq 1$.

Assume $\left(K_{X}+A\right)^{2}=1$. Then $-K_{X} \cdot\left(K_{X}+A\right)$ is odd, so we only have to worry about $-K_{X}$. $\left(K_{X}+A\right)=1$. Recall that $K_{X}+A$ is free and big, therefore by Kawamata-Viehweg Theorem, $h^{1}\left(2 K_{X}+A\right)=h^{2}\left(2 K_{X}+A\right)=0$. Since $\left(2 K_{X}+A\right) \cdot\left(K_{X}+A\right)=0$, by Riemann-Roch it follows that $h^{0}\left(2 K_{X}+A\right)=1$. On the other hand, since $K_{X}^{2}=\left(K_{X}+A\right)^{2}=1$, then $A \cdot\left(2 K_{X}+A\right)=0$, hence $A=-2 K_{X}$. This is excluded because at this point we are assuming $A^{2} \geq 5$.

Assume $\left(K_{X}+A\right)^{2}=2$. Then $-K_{X} \cdot\left(K_{X}+A\right)$ is even, so we only have to worry about $-K_{X} \cdot\left(K_{X}+A\right)=2$. Note that $A \cdot\left(K_{X}+A\right)=4$. Then by Riemann-Roch $\left|K_{X}+A\right|$ maps $X$ as double cover of $\mathbf{P}^{2}$. Then either $K_{X}^{2}=8$, which is excluded by hypothesis or $K_{X}^{2}=2$, which is excluded by assumption.

Assume $\left(K_{X}+A\right)^{2}=3$. Then $-K_{X} \cdot\left(K_{X}+A\right)$ is odd, so we only have to worry about $-K_{X}$. $\left(K_{X}+A\right)=1$. This cannot happen by Hodge Index Theorem.

Assume $\left(K_{X}+A\right)^{2}=4$. Then $-K_{X} \cdot\left(K_{X}+A\right)$ is even, so we only have to worry about $-K_{X} \cdot\left(K_{X}+A\right)=2$. First we see that $\left(2 K_{X}+A\right)$ is effective. Indeed, $\left(2 K_{X}+A\right) \cdot\left(K_{X}+A\right)=2$, then by Kawamata-Viehweg Theorem and Riemann-Roch, $h^{0}\left(2 K_{X}+A\right)=2$. Then $h^{2}\left(3 K_{X}+A\right)=$ $h^{0}\left(-2 K_{X}-A\right)=0$. We also have that $\left(3 K_{X}+A\right) \cdot\left(2 K_{X}+A\right)=0$. Then by Riemann-Roch $3 K_{X}+A$ is effective. On the other hand $A \cdot\left(3 K_{X}+A\right) \leq 0$, hence $A \cdot\left(3 K_{X}+A\right)=0$ and $A=-3 K_{X}$.

We deal now with the case $A^{2} \leq 4$. By Lemma 1.7 , if $A^{2}=1$, then $A=-K_{X}$, which is excluded by assumption. If $A^{2} \geq 2$, by Hodge Index Theorem $-K_{X} \cdot A \geq 2$, hence by [Hb1], Theorem III.1.a $A$ is base point free. If $A^{2}=2$, then either $X=\mathbf{P}^{1} \times \mathbf{P}^{1}$, which is excluded by hypothesis or $K_{X}^{2}=2$ and $A=-K_{X}$, which is excluded by assumption. 
If $A^{2}=3$, by Hodge Index Theorem and because $-K_{X} \cdot A$ must be odd $-K_{X} \cdot A \geq 3$, hence by Theorem 1.3, $A$ is very ample. Then $X$ is a Del Pezzo cubic surface in $\mathbf{P}^{3}$ and $A=-K_{X}$ or a rational normal scroll in $\mathbf{P}^{4}$. Both possibilities are excluded either by assumption or by hypothesis.

Finally let $A^{2}=4$. Again by Hodge Index Theorem $-K_{X} \cdot A \geq 2$ and $A$ is base-point-free. If $-K_{X} \cdot A \geq 3, A$ is actually very ample by Theorem 1.3. However the only linearly normal smooth surfaces of degree 4 are K3 surfaces in $\mathbf{P}^{3}$, the Del Pezzo surface in $\mathbf{P}^{4}$ and the Veronese Surface and the rational normal scrolls in $\mathbf{P}^{5}$. Then the only possibility left to study is $A^{2}=4$ and $-K_{X} \cdot A=2$. Then $A \cdot\left(2 K_{X}+A\right)=0$. On the other hand $h^{2}\left(2 K_{X}+A\right)=h^{0}\left(-K_{X}-A\right)=0$, because $A \cdot\left(-K_{X}-A\right)=-2$. Then it follows by Riemann-Roch that $2 K_{X} \cdot A_{1}$ is effective, so $A=-2 K_{X}$.

Before stating and proving Theorems 1.23 and 1.24, which are consequences of Propositions 1.6, 1.9 and 1.10 it is important to know that these propositions are sharp. We do this by means of the following series of examples. Then these examples will also imply the optimality of our theorems on rational surfaces.

Example 1.11. $K_{X}^{2}=9, A$ ample, $-K_{X} \cdot A=3, K_{X}+3 A$ not ample.

Of course the only rational surface with $K_{X}^{2}=9$ is $X=\mathbf{P}^{2}, A=\mathcal{O}_{\mathbf{P}^{2}}(1)$ attains the bound $-K_{X} \cdot A \geq 3$ and $K_{X}+3 A=\mathcal{O}_{\mathbf{P}^{2}}$, hence not very ample.

Example 1.12. $K_{X}^{2}=8, A$ ample, $-K_{X} \cdot A=e+4, K_{X}+2 A$ not ample.

The rational surfaces with $K_{X}^{2}=8$ are the Hirzebruch surfaces $\mathbf{F}_{e}$. With these examples we show that the bounds computed in Proposition 1.9 for $-K_{X} \cdot A$ in terms of $e$ are sharp. If $X=\mathbf{F}_{e}$, let $C_{0}$ be the minimal section and $f$ a fiber. The divisor $A=C_{0}+(e+1) f$ is ample and $-K_{X} \cdot A$ achieves the bound $e+4$. Moreover $K_{X}+2 A=e f$ is free but not ample, hence $A$ provides an example of $K_{X}+2 A$ not being very ample.

Example 1.13. $3 \leq K_{X}^{2} \leq 7, A$ ample, $-K_{X} \cdot A=K_{X}^{2}, K_{X}+A$ not ample.

We obtain $X$ with $K_{X}^{2}=9-i$ by blowing up $\mathbf{P}^{2}$ at $2 \leq i \leq 6$ points, which we choose in sufficiently general position (not 3 of them on a line, not 6 of them on a conic). This is a Del Pezzo surface and $-K_{X}$ is very ample (cf. [Ht], Theorem V.4.6). Then $\left(X,-K_{X}\right)$ achieves the bound $A^{2} \geq K_{X}^{2}$ and also provides an example where $K_{X}+A$ is not very ample.

Example 1.14. $K_{X}^{2}=2, A$ ample, $-K_{X} \cdot A=K_{X}^{2}$ and $K_{X}+2 A$ not very ample.

Let $p: X \longrightarrow \mathbf{P}^{2}$ be the double cover of $\mathbf{P}^{2}$ ramified along a smooth quartic. Such an $X$ is rational, $K_{X}=p^{*}\left(\mathcal{O}_{\mathbf{P}^{2}}(-1)\right)$ and $-K_{X}=p^{*}\left(\mathcal{O}_{\mathbf{P}^{2}}(1)\right)$. Therefore $K_{X}^{2}=2$ and, since $p$ is finite, $-K_{X}$ is ample and base-point-free. In fact, $p$ is induced by the morphism of the complete anticanonical linear series. We remark that, actually, a rational surface $X$ with $K_{X}^{2}=2$ and with anticanonical divisor ample is a double cover of $\mathbf{P}^{2}$ as the one described above. This follows from Riemann-Roch. Thus the anticanonical divisor on such a surface $X$ attains the bound $A^{2} \geq K_{X}^{2}$.

Such a surface $X$ can also be found embedded in $\mathbf{P}^{6}$. Indeed, $-2 K_{X}$ is very ample, and embeds $X$ as a degree 8, sectional genus 6, smooth surface in $\mathbf{P}^{6}$ (see [BS], Example 10.2.4).

In addition $A=-K_{X}$ provides an example of $K_{X}+2 A$ not being very ample.

Example 1.15. $K_{X}^{2}=1, A$ ample, $-K_{X} \cdot A=1, K_{X}+3 A$ not very ample.

An example of a surface $X$ with $K_{X}^{2}=1$ and $-K_{X}$ ample can be found in [BS], Example 10.4.3. Indeed there exists a smooth rational surface with $K_{X}^{2}=1$ and $-K_{X}$ ample. This surface is embedded by $\left|-3 K_{X}\right|$ as a degree 9 , sectional genus 4 smooth surface in $\mathbf{P}^{6}$. Then $\left(X,-K_{X}\right)$ 
achieves the bound $A^{2} \geq K_{X}^{2}$. In addition $A=-K_{X}$ provides an example of $K_{X}+3 A$ not being very ample. Indeed, $-2 K_{X}$ is not very ample as its complete linear series induces a double cover of quadric cone in $\mathbf{P}^{3}$.

Example 1.16. $-1 \leq K_{X}^{2} \leq 8, A$ ample, $-K_{X} \cdot A=K_{X}^{2}+2, K_{X}+A$ not ample.

The family of examples we consider now are conic bundles. Let us fix $n=K_{X}^{2},-1 \leq n \leq 8$. The examples are constructed from the pair $\left(Y=\mathbf{F}_{e}, 2 C_{0}+m f\right)$, where $m=e+3,0 \leq e \leq 2, C_{0}$ is the minimal section of $Y$ and $f$ is a fiber of $Y$. Let $C$ be a smooth anticanonical divisor on $Y$. Let $l=8-n$. We choose $\Sigma=\left\{p_{1}, \ldots, p_{l}\right\}, l$ distinct points on $C$ lying on different fibers of $Y$. Let $\pi: X \longrightarrow Y$ be the blowing-up of $Y$ along $\Sigma$ and let $E_{1}, \ldots, E_{l}$ be the exceptional divisors. Let $A=\pi^{*}\left(2 C_{0}+m f\right)-E_{1}-\cdots-E_{l}$. By the choice of $m, K_{X}+A=\pi^{*} f$. Hence $K_{X}+A$ is not ample and $-K_{X} \cdot A=K_{X}^{2}+2$. We see now that $A$ is ample using Nakai-Moishezon's criterion. Firstly, as $n \geq-1, A^{2} \geq 3$. Secondly, it is clear that $A \cdot E_{i}=1$, for all $1 \leq i \leq l$. Finally we will check that the intersection of $A$ with any irreducible non-exceptional curve $T$ on $X$ is strictly positive. Let $D=\pi(T)$ and let $D \sim a C_{0}+b f$. Let $m_{1}, \ldots, m_{l}$ be the multiplicities of $D$ at $p_{1}, \ldots, p_{l}$. Then $A \cdot T=\left(2 C_{0}+m f\right) \cdot D-m_{1}-\cdots-m_{l}$. Hence we want $m_{1}+\cdots+m_{l}<\left(2 C_{0}+m f\right) \cdot D=3 a+2 b-a e$. We first consider the case when $C$ and $D$ intersect properly. Since $p_{1}, \ldots, p_{l} \in C, m_{1}+\cdots+m_{l} \leq C \cdot D$. Therefore $m_{1}+\cdots+m_{l} \leq 2 a+2 b-a e$. Then if $a>0$ we are done. If $a=0$, then $D=f$. Since we have chosen $p_{1}, \ldots, p_{l}$ in different fibers in this case $m_{1}+\cdots+m_{l}=1<2=3 a+2 b-a e$, and we are also done. Now consider the case when $C$ and $D$ do not intersect properly. Since both $C$ and $D$ are irreducible, $C=D$. In this case $m_{1}+\cdots+m_{l}=l$, for $C$ is smooth, and $a=2$ and $b=e+2$. Then $3 a+2 b-a e=10$ and we are done if $l<10$. The latter happens because $n \geq-1$.

Example 1.17. $-2 \leq K_{X}^{2} \leq 8, A$ ample, $-K_{X} \cdot A=K_{X}^{2}+3$ and $K_{X}+A$ not ample.

Let $Y=\mathbf{F}_{1}$, let $C_{0}$ the minimal section, let $f$ be a fiber and let $C$ be a smooth irreducible anticanonical curve. Let $\pi: X \longrightarrow Y$ the blowing up of $Y$ at $\Sigma=\left\{p_{1}, \ldots, p_{l}\right\}$, where $p_{1}, \ldots, p_{l}$ are distinct points of $Y$ on $C$ away from $C_{0}$ and $0 \leq l \leq 10$. Let $E_{1}, \ldots, E_{l}$ be the exceptional divisors lying over $p_{1}, \ldots, p_{l}$ respectively. Let $A=\pi^{*}\left(3 C_{0}+4 f\right)-E_{1}-E_{2}-\cdots-E_{l}$. We claim that $A$ is ample, that $-K_{X} \cdot\left(K_{X}+A\right)=3$ and that $K_{X}+A$ is not ample. The latter is clear, since $K_{X}+A=\pi^{*}\left(C_{0}+f\right)$. From this it also follows that $-K_{X} \cdot\left(K_{X}+A\right)=3$. We check finally the ampleness of A using Nakai-Moishezon's criterion. On the one hand $A^{2}=15-l \geq 5$, since $l \leq 10$. Clearly $A \cdot E_{i}=1$. Let $T$ be now a nonexceptional irreducible curve on $X$, and let $D=\pi(T)$. Let $D \sim a C_{0}+b f$ and let $m_{1}, \ldots, m_{l}$ be the multiplicities of $D$ at $p_{1}, \ldots, p_{l}$. Then $A \cdot T=\left(3 C_{0}+4 f\right) \cdot D-m_{1}-\cdots-m_{l}=a+3 b-m_{1}-\cdots-m_{l}$, so we want $m_{1}+m_{2}+\cdots+m_{l}<a+3 b$. We distinguish several cases: first we consider the case when $C$ and $D$ intersect properly. Then $m_{1}+\cdots+m_{l} \leq C \cdot D=a+2 b$ since every $p_{i}$ lies on $C$. We consider two subcases: $b>0$ or $b=0$. If the former, $a+2 b<a+3 b$ and we are done. If the latter $T=C_{0}$ and $m_{1}=\cdots=m_{l}=0$ by our choice of $p_{1}, \ldots, p_{l}$. Since $0=m_{1}+\cdots+m_{l}<1$ we are also done. The only case left is when $C$ and $D$ do not intersect properly. Since both $C$ and $D$ are irreducible, $C=D$. Since $C$ is smooth, $m_{1}+\cdots+m_{l}=l$. On the other hand $a+3 b=11$ in this case, and, since $l \leq 10$, we are done.

Example 1.18. $K_{X}^{2}=0, A$ ample, $-K_{X} \cdot A=1, K_{X}+2 A$ not very ample.

We find a surface with $K_{X}^{2}=0$ with an ample line bundle $A$ such that $-K_{X} \cdot A=1$ and $K_{X}+2 A$ is not very ample. Let $Y=\mathbf{P}^{2}$. We consider a set $\Sigma \subset Y$ of 9 distinct points being the complete intersection of 2 cubics, and neither 3 of them on a line nor 6 of them on a conic. Let $X$ be the blowing up of $Y$ along $\Sigma$. Then $-K_{X}$ is base-point-free, $h^{0}\left(-K_{X}\right)=2$, and all $C \in\left|-K_{X}\right|$ are irreducible curves. Therefore $\left|-K_{X}\right|$ turns $Y$ into an elliptic fibration $\varphi: X \longrightarrow \mathbf{P}^{1}$ with irreducible fibers and 
at least 9 sections, namely, the 9 exceptional divisors. Let $E$ be one of the exceptional divisors and let $F$ be a fiber of $\varphi$. Then $A=E+2 F$ is ample. Indeed, we use Nakai-Moishezon's criterion. The self-intersection $(E+2 F)^{2}=E^{2}+4 E \cdot F=3$. If $F^{\prime}$ is a fiber of $\varphi,(E+2 F) \cdot F^{\prime}=1$. Let $C$ be an irreducible curve on $X$, and not a fiber. Then either $C=E$, in which case $(E+2 F) \cdot C=1$ or $(E+2 F) \cdot C=E \cdot C+2 F \cdot C \geq 2$. Now we see that $-K_{X} \cdot A=1$ and that $K_{X}+2 A$ is not very ample. It is clear that $-K_{X} \cdot A=1$. We see now that $K_{X}+2 A$ is not very ample. Indeed, let $C \in\left|-K_{X}\right|$. Then $C$ has arithmetic genus 1 ; however $K_{X}+2 A=-3 K_{X}+2 E$, hence $\left(K_{X}+2 A\right) \cdot C=2$. Since the arithmetic genus of $C$ is $1, K_{X}+2 A$ is not very ample. Note that $A^{\prime}=E+n F$ for some $n \geq 2$ satisfies as well that $-K_{X} \cdot A^{\prime}=1$ and $K_{X}+2 A^{\prime}$ is not very ample.

Example 1.19. $K_{X}^{2}<0$ odd, $A$ ample, $-K_{X} \cdot A=1$ and $K_{X}+A$ not ample.

Given $n$ odd number strictly smaller than 0 , we find a surface $X$ with $K_{X}^{2}=n$ and an ample line bundle $A$ on $X$ such that $-K_{X} \cdot A=1$. Let $Y=\mathbf{F}_{0}$, let $l=8-n$. Let $C$ be a smooth anticanonical curve on $Y$ and $f_{1}$ and $f_{2}$ be two lines each belonging to one ruling of $\mathbf{F}_{0}$. Let $\Sigma=\left\{p_{1}, \ldots, p_{l}\right\}$ be $l$ distinct points on $C$ chosen so that not two of them belong to a line of $\mathbf{F}_{0}$. Let $\pi: X \longrightarrow Y$ be the blowing up of $Y$ along $\Sigma$ and let $E_{i}$ be the exceptional divisor over $p_{i}$. Let $k=\frac{l-3}{2}$ and let $A=$ $\pi^{*}\left(\mathcal{O}_{\mathbf{F}_{0}}\left(2 f_{1}+k f_{2}\right)-E_{1}-\cdots-E_{l}\right)$. We see that $-K_{X} \cdot A=\left(2 f_{1}+2 f_{2}\right) \cdot\left(2 f_{1}+k f_{2}\right)+E_{1}^{2}+\cdots+E_{l}^{2}=1$. Moreover $K_{X}+A=\pi^{*}\left((k-2) f_{2}\right)$, and therefore it is not ample. Now we will see that $A$ is ample using Nakai-Moishezon's criterion. First $A^{2}=l-6 \geq 3$. Now we see the intersection of $A$ with irreducible curves on $X$. The intersection of $A$ with each $E_{i}$ is 1 . Let $T$ be an irreducible curve which is not an exceptional divisor and let $D=\pi(T)$. Let $D \sim a f_{1}+b f_{2}$. Let $m_{1}, \ldots, m_{l}$ be the multiplicities of $D$ at $p_{1}, \ldots, p_{l}$. Then $A \cdot T=\left(2 f_{1}+k f_{2}\right) \cdot D-m_{1}-\cdots-m_{l}$. Hence we want to see that $m_{1}+\cdots+m_{l}<k a+2 b$. Since $p_{1}, \ldots, p_{l} \in C, m_{1}+\cdots+m_{l} \leq C \cdot D$ if $C$ and $D$ intersect properly. Then $m_{1}+\cdots+m_{l} \leq 2 a+2 b$. Since $n \leq-1, k \geq 3$, hence if $a \geq 1,2 a+2 b<k a+2 b$ and we are done. If $a=0$, then $D=f_{2}$ and by our choice of $p_{1}, \ldots, p_{l}, m_{1}+\cdots+m_{l}=1<2=k a+2 b$ and we are also done. Now suppose that $C$ and $D$ do not intersect properly. Since both $C$ and $D$ are irreducible, $C=D$ and $a=b=2$. In this case $m_{1}+\cdots+m_{l}=l$, for $C$ is smooth. Since $k=\frac{l-3}{2}$, $l<2 k+4$.

Example 1.20. $K_{X}^{2}<0$ even, $A$ ample, $-K_{X} \cdot A=1$ and $K_{X}+A$ not ample.

Given $n$ even number strictly smaller than 0 , we find a surface $X$ with $K_{X}^{2}=n$ and an ample line bundle $A$ on $X$ such that $-K_{X} \cdot A=1$. Let $Y=\mathbf{F}_{0}$ and let $l=8-n$. Let $C$ be a smooth anticanonical curve on $Y$ and $f_{1}$ and $f_{2}$ be two lines each belonging to one ruling of $\mathbf{F}_{0}$. Let $\Sigma=\left\{p_{1}, \ldots, p_{l}\right\}$ be $l$ distinct points on $C$ chosen so that not two of them belong to a line of $\mathbf{F}_{0}$. Let $\pi: X \longrightarrow Y$ be the blowing up of $Y$ along $\Sigma$ and let $E_{i}$ be the exceptional divisor over $p_{i}$. Let $k=\frac{l-4}{2}$ and let $A=\pi^{*}\left(\mathcal{O}_{\mathbf{F}_{0}}\left(3 f_{1}+k f_{2}\right)-2 E_{1}-E_{2}-\cdots-E_{l}\right.$. Then $-K_{X} \cdot A=$ $\left(2 f_{1}+2 f_{2}\right) \cdot\left(3 f_{1}+k f_{2}\right)+2 E_{1}^{2}+E_{2}^{2}+\cdots+E_{l}^{2}=1$. In addition $K_{X}+A=\pi^{*}\left(f_{1}+(k-2) f_{2}\right)-E_{1}$. Therefore $K_{X}+A$ is not ample, since its intersection with the strict transform of one of the lines passing through $p_{1}$ is 0 . We see now that $A$ is ample using Nakai-Moishezon's criterion. First $A^{2}=2 l-15 \geq 5$. Now we see the intersection of $A$ with the irreducible curves on $X$. The intersection of $A$ with $E_{1}$ is 2 and with the other exceptional divisors is 1 . Let $T$ be an irreducible curve which is not an exceptional divisor and let $D=\pi(T)$. Let $D \sim a f_{1}+b f_{2}$. Let $m_{1}, \ldots, m_{l}$ the multiplicities of $D$ at $p_{1}, \ldots, p_{l}$. Then $A \cdot T=\left(3 f_{1}+k f_{2}\right) \cdot D-2 m_{1}-m_{2}-\cdots-m_{l}$. Hence we want to see that $2 m_{1}+m_{2}+\cdots+m_{l}<k a+3 b$. First we suppose that $C$ and $D$ intersect properly. Since $p_{1}, \ldots, p_{l} \in C$, then $m_{1}+\cdots+m_{l} \leq C \cdot D=2 a+2 b$. We distinguish now two cases. First, if $m_{1} \leq a$, then $2 m_{1}+m_{2}+\cdots+m_{l} \leq 3 a+2 b$. Since $n \leq-2, k \geq 3$. Then if $b \geq 1$, $3 a+2 b<k a+3 b$ and we are done. If $b=0$, then $D \sim f_{1}$ and $a=1$. By the choice of $p_{1}, \ldots, p_{l}$, 
$2 m_{1}+m_{2}+\cdots+m_{l} \leq 2<3 \leq k a+3 b$. Second, if $m_{1}>a$, then $D$ passes through $p_{1}$ and $D \sim f_{2}$. Then, because of the choice of $p_{1}, \ldots, p_{l}, 2 m_{1}+m_{2}+\cdots+m_{l} \leq 2<3=k a+3 b$. Now we suppose that $C$ and $D$ do not intersect properly. Since $C$ and $D$ are irreducible, then $C=D$, and $a=b=2$. In this case $2 m_{1}+m_{2}+\cdots+m_{l}=l+1$, for $C$ is smooth. Then we are done if $l+1<2 k+6=k a+3 b$. This occurs because $k=\frac{l-4}{2}$.

The lower bounds for $-K_{X} \cdot A$ obtained in Proposition 1.9 and Proposition 1.10 combined with Theorem 1.3 yield several results. The following generalizes a well known result, namely, the equivalence of the notion of ampleness and very ampleness for $\mathbf{P}^{2}$ and for Hirzebruch surfaces (cf. [Ht] , Corollary V.2.18). The result we get is actually stronger than this classical result since we obtain the equivalence of ampleness and certain $N_{p}$ property for certain anticanonical surfaces:

Theorem 1.21. Let $X$ be a rational surface such that $d=K_{X}^{2} \geq 3$. Let $A$ be a line bundle on $X$. The following are equivalent:

1) A is ample;

2) $A$ is very ample;

3) A satisfies property $N_{0}$. More precisely:

- If $3 \leq K_{X}^{2} \leq 7$, then $A$ satisfies property $N_{d-3}$ if and only if $A$ is ample and $A$ satisfies property $N_{d-1}$ if and only if $A$ is ample different from $-K_{X}\left(-K_{X}\right.$ satisfies $N_{d-3}$ but not $\left.N_{d-2}\right)$.

- If $X=\mathbf{F}_{e}$, then $A$ satisfies property $N_{e+1}$ if and only if $A$ is ample.

- If $X=\mathbf{P}^{2}$, then $A$ satisfies property $N_{0}$ if and only if $A$ is ample.

In addition, if $K_{X}^{2}=2$, then $A$ satisfies property $N_{1}$ if and only if $A$ is an ample line bundle different form $-K_{X}$.

In the next result we show that very ampleness and projective normality are equivalent for anticanonical surfaces:

Proposition 1.22. Let $X$ be an anticanonical surface. A line bundle on $X$ is very ample if and only if it satisfies property $N_{0}$.

Proof. Let $L$ be very ample and let $C$ be smooth curve in $|L|$. Since $-K_{X} \otimes \mathcal{O}_{C}$ is effective, $-K_{X} \cdot L \geq 3$, otherwise $L \otimes \mathcal{O}_{C}$ would not be very ample. Then $L$ satisfies property $N_{0}$ by Theorem 1.3.

We state and show now the result already announced dealing with line bundles of the form $K_{X}+A_{1}+\cdots+A_{n}$, with $A_{1}, \ldots, A_{n}$ ample. It follows from Theorem 1.3 and Propositions 1.6, 1.9 and 1.10 .

Theorem 1.23. Let $X$ be an anticanonical surface. Let $A_{1}, \ldots, A_{n}$ be ample line bundles on $X$. If $n \geq p+4$, then $L=K_{X}+A_{1}+\cdots+A_{n}$ satisfies property $N_{p}$. This bound is achieved for $A_{i}=A$ and $(X, A)$ as in Example 1.15 .

More precisely:

1) If $X=\mathbf{P}^{2}$ and $n \geq\left\lceil\frac{p}{3}\right\rceil+4$, then $L$ satisfies property $N_{p}$. The bound is achieved for $A_{i}=\mathcal{O}_{\mathbf{P}^{2}}(1)$ and $p$ multiple of 3 .

2) If $K_{X}^{2}=8$ and $n \geq\left\lceil\frac{p+3}{4}\right\rceil+2$, then $L$ satisfies property $N_{p}$. The bound is achieved for $X=\mathbf{F}_{0}$, $A_{i}=C_{0}+f$ and $p \equiv 1(4)$. More precisely, if $X=\mathbf{F}_{e}$ and $n \geq \max \left(3,\left\lceil\frac{p+11}{e+4}\right\rceil\right)$, then $L$ satisfies property $N_{p}$. The bound is achieved for instance for $A_{i}=C_{0}+(e+1) f$ and for $p \equiv-11(e+4)$.

3) If $1 \leq K_{X}^{2} \leq 7$ and $n \geq\left\lceil\frac{p+3}{K^{2}}\right\rceil+1$, then $L$ satisfies property $N_{p}$. The bound is achieved for instance for $\left(X, A_{i}\right)$ as in Examples 1.13, 1.14 and 1.15, and for $p \equiv-3\left(K_{X}^{2}\right)$. 
4) If $1 \leq K_{X}^{2} \leq 7, A_{i} \neq-K_{X}, A_{i} \neq-2 K_{X}$ if $K_{X}^{2}=1$ and $n \geq\left\lceil\frac{p+K_{X}^{2}+3}{K_{X}^{2}+2}\right\rceil$, then $L$ satisfies property $N_{p}$. The bound is achieved for $\left(X, A_{i}\right)$ as in Example 1.16, and for $p+K_{X}^{2} \equiv-3\left(K_{X}^{2}+2\right)$.

5) If $2 \leq K_{X}^{2} \leq 7, A_{i} \neq-K_{X}, A_{i} \neq-2 K_{X}$ when $K_{X}^{2}=2, A_{i} \neq-2 K_{X},-3 K_{X}$ when $K_{X}^{2}=1$, it does not happen that $K_{X}+A_{i}$ is base-point-free, $A_{i}$ is very ample and $\left(X, A_{i}\right)$ is a conic fibration under $\left|K_{X}+A_{i}\right|$, and $n \geq \max \left(2,\left\lceil\frac{p+K_{X}^{2}+3}{K_{X}^{2}+3}\right\rceil\right)$, then $L$ satisfies property $N_{p}$. The bound is achieved for $\left(X, A_{i}\right)$ as in Example 1.17, and for $p+K_{X}^{2} \equiv-3\left(K_{X}^{2}+3\right)$.

6) If $-1 \leq K_{X}^{2} \leq 1$ and $n \geq p+3+K_{X}^{2}$, then $L$ satisfies property $N_{p}$. The bound is sharp for $\left(X, A_{i}\right)$ as in Examples 1.15, 1.18 and 1.19 .

7) If $K_{X}^{2} \leq-2$ and $n \geq \max \left(2, p+3+K_{X}^{2}\right)$, then $L$ satisfies property $N_{p}$. The bound is sharp for $\left(X, A_{i}\right)$ as in Examples 1.19 and 1.20.

The next result we will prove is Theorem 1.24, which is an $N_{p}$ result in the same flavor of Reider's theorem for base-point-freeness and very ampleness. Theorem 1.24 shows that when $K_{X}^{2} \geq 1$, a high self-intersection number for $L$ implies by itself property $N_{p}$ for $K_{X}+L$ for a large value of $p$. This behavior is in contrast with the behavior that can be observed in surfaces of Kodaira dimension 0.

Theorem 1.24. Let $X$ be a rational surface. Let $L$ be a line bundle such that

1) $L \cdot C \geq 3$ for any curve $C$ on $X$ and $L^{2} \geq 10$ or

1') $K_{X}+L$ is very ample.

If $K_{X}^{2} \geq 1$ :

2a) Let $L^{2} \geq(p+3)^{2}+1$.

If $K_{X}^{2} \geq 1$ and $L$ is not a multiple of $-K_{X}$ when $K_{X}^{2}=1$ :

2b) Let $L^{2} \geq(p+3)^{2}-1$.

If $K_{X}^{2} \leq 0$ :

2c) Let $-K_{X} \cdot L \geq p+3$.

Then $K_{X}+L$ satisfies property $N_{p}$.

To prove Theorem 1.24 we will use Theorem 1.3, Proposition 1.22 and the technical lemma 1.25. The lemma is proven by contradiction and the argument connects property $N_{p}$ with termination of adjunction. In particular it shows that a failure of certain property $N_{p}$ to hold results in non termination of adjunction.

Lemma 1.25 . Let $X$ be a rational surface with $K_{X}^{2}>0$ and not isomorphic to $\mathbf{P}^{2}$. Let $L$ be a line bundle on $X$ such that $K_{X}+L$ is effective. Let $p \geq 1$ if $K_{X}^{2} \leq 7$ and $p \geq 2$ if $K_{X}^{2}=8$. If $L^{2} \geq(p+3)^{2}-1$ and $L$ is not a positive multiple of $-K_{X}$ when $K_{X}^{2}=1$, then $-K_{X} \cdot L \geq p+3+K_{X}^{2}$.

Proof. Assume that, $L$ is not a positive multiple of $-K_{X}$, or $K_{X}^{2} \geq 2$. We will prove the result by way of contradiction. Assuming that $-K_{X} \cdot\left(K_{X}+L\right) \leq p+2$, we will prove by induction that $m K_{X}+L$ is effective for all $m \geq 2$. This contradicts the termination of adjunction on a surface of Kodaira dimension $-\infty$.

If $m=1, K_{X}+L$ is effective by hypothesis. Let us see now that $2 K_{X}+L$ is effective. Note that $L \neq-K_{X}$ because $L^{2} \geq 15$ and $K_{X}^{2} \leq 8$. Then, since $K_{X}+L$ is effective, $-K_{X}-L$ is not effective. Then by Riemann-Roch

$$
h^{0}\left(2 K_{X}+L\right) \geq \frac{1}{2}\left(2 K_{X}+L\right)\left(K_{X}+L\right)+1 .
$$


Therefore we need to see that $\left(2 K_{X}+L\right)\left(K_{X}+L\right)>-2$. This inequality is equivalent to

$$
L^{2}+2 K_{X}^{2}+1 \geq 3\left(-K_{X} \cdot L\right)
$$

Since by assumption $-K_{X} \cdot L \leq p+2+K_{X}^{2}$, it suffices to check that $L^{2} \geq 3(p+2)+K_{X}^{2}-1$. Then it is enough to see that $(p+3)^{2} \geq 3 p+6+K_{X}^{2}$. This last inequality is equivalent to $p^{2}+3 p+3-K_{X}^{2} \geq 0$, which holds for all $p \geq 1$ if $K_{X}^{2} \leq 7$ and for all $p \geq 2$ if $K_{X}^{2}=8$.

Let $m \geq 2$. Now we assume $m K_{X}+L$ is effective, and we will show $(m+1) K_{X}+L$ is effective. First consider the case when $K_{X}^{2}=1$ and $L$ is not multiple of $-K_{X}$. Since $m K_{X}+L$ is effective, $h^{0}\left(-m K_{X}-L\right)=0$. Then by Riemann-Roch

$$
h^{0}\left((m+1) K_{X}+L\right) \geq \frac{1}{2}\left((m+1) K_{X}+L\right)\left(m K_{X}+L\right)+1 .
$$

Thus we need to see that $\left((m+1) K_{X}+L\right)\left(m K_{X}+L\right) \geq-1$. This is equivalent to

$$
L^{2}+m(m+1) K_{X}^{2}+1 \geq(2 m+1)\left(-K_{X} \cdot L\right) .
$$

Since by assumption $-K_{X} \cdot L \leq p+2+K_{X}^{2}$, it suffices to check that

$$
L^{2} \geq(2 m+1)(p+2)+\left(-m^{2}+m+1\right) K_{X}^{2}-1 .
$$

Recall that $K_{X}^{2}=1$. Then, in order to check (1.25.1) it is enough to show that

$$
(p+3)^{2}-1 \geq(2 m+1)(p+2)-m^{2}+m .
$$

This last inequality is equivalent to

$$
(p-m)^{2}+5(p-m)+6 \geq 0 .
$$

This holds for all integers $p$ and $m$.

Now we assume that $K_{X}^{2} \geq 2$. Since $m K_{X}+L$ is effective, $h^{0}\left(-m K_{X}-L\right) \leq 1$. Then by Riemann-Roch

$$
h^{0}\left((m+1) K_{X}+L\right) \geq \frac{1}{2}\left((m+1) K_{X}+L\right)\left(m K_{X}+L\right) .
$$

Thus we need to see that $\left((m+1) K_{X}+L\right)\left(m K_{X}+L\right)>0$. This is equivalent to

$$
L^{2}+m(m+1) K_{X}^{2}>(2 m+1)\left(-K_{X} \cdot L\right)
$$

Since by assumption $-K_{X} \cdot L \leq p+2+K_{X}^{2}$, it suffices to check that

$$
L^{2}>(2 m+1)(p+2)+\left(-m^{2}+m+1\right) K_{X}^{2} .
$$

Since $m \geq 2,-m^{2}+m+1<0$. Then, in order to check (1.25.2) it is enough to show that

$$
(p+3)^{2}-1>(2 m+1)(p+2)-2\left(m^{2}-m-1\right) .
$$

This last inequality is equivalent to

$$
p^{2}+(5-2 m) p+\left(2 m^{2}-6 m+4\right)>0 .
$$


This holds for any integer $p \geq 1$ and any $m \geq 2$.

Summing up, we have just shown that, under the hypothesis of the proposition and with the additional assumption that $-K_{X} \cdot L \leq p+2+K_{X}^{2}, m K_{X}+L$ is effective for all $m \geq 2$. As pointed out before, this is a contradiction. Therefore $-K_{X} \cdot L \geq p+3+K_{X}^{2}$, as wished.

Remark 1.26. Lemma 1.25 follows from Hodge Index Theorem for some values of $p$ and $K_{X}^{2}$ but not for all.

(1.27) Proof of Theorem 1.24. By hypothesis 1') or by 1) and Reider's Theorem it follows that $K_{X}+L$ is very ample. Therefore by Proposition $1.22 K_{X}+L$ satisfies property $N_{0}$. If $X=\mathbf{P}^{2}$, it follows clearly from $2 \mathrm{~b}$ ) that $-K_{X} \cdot\left(K_{X}+L\right) \geq p+3$ if $p \geq 2$. Then by Theorem $1.3 K_{X}+L$ satisfies property $N_{p}$ (if $L$ is such that $L^{2}=16$, then one could say that $K_{X}+L$ satisfies $N_{\infty}$ ). We consider now $X=\mathbf{F}_{e}$. If $p \geq 2$, by Lemma $1.25,2 \mathrm{~b}$ ) and Theorem $1.3 \mathrm{~L}$ satisfies property $N_{p}$. If $p=1$ the result also follows because if $K_{X}+L$ is very ample it can be seen that $L^{2} \geq 18$. Now if $K_{X}^{2}=1$ and $L=m\left(-K_{X}\right)$, then $\left.2 \mathrm{a}\right)$ implies $m \geq p+4$. Therefore it follows by Theorem 1.3 that $K_{X}+L$ satisfies $N_{p}$. If $L$ is not a multiple of $-K_{X}$ or if $K_{X}^{2} \geq 2$ and $X \neq \mathbf{P}^{2}$, then it follows from 2b) and Lemma 1.25 that $-K_{X} \cdot\left(K_{X}+L\right) \geq p+3$. Thus $K_{X}+L$ satisfies property $N_{p}$ by Theorem 1.3. Finally if $K_{X}^{2} \leq 0$, by 2c) $-K_{X} \cdot\left(K_{X}+L\right) \geq p+3$. Thus $K_{X}+L$ satisfies property $N_{p}$ by Theorem 1.3.

Remark 1.28 Theorem 1.24 is optimal. To see the optimality when $2 \mathrm{a}$ ) is assumed, take $X$ as in Example 1.15 and $L=(p+4)\left(-K_{X}\right)$. To see the optimality when $\left.1^{\prime}\right)$ and $\left.2 \mathrm{~b}\right)$ are assumed take $A$ as in Example 1.16,e $=0, l=6$ and $L=-K_{X}+A$. Then $K_{X}+L$ satisfies property $N_{1}$ but not $N_{2}$ by Theorem 1.3 and $L^{2}=16$. On the other hand if $K_{X}^{2} \leq 0$, assuming only hypothesis 1 ) and $2 \mathrm{a})$ or 1') and 2a) do not suffice. This can be seen taking $(X, A)$ as in Example 1.18 and $L=3 A$. Indeed $K_{X}+L$ satisfies property $N_{0}$ but not $N_{1}$; however, $L^{2}=27>(2+3)^{2}+1$.

We end this section by showing the relation between the property $N_{p}$ satisfied by a line bundle $L$ and the termination of ampleness for $m K_{X}+L$.

Theorem 1.29. Let $X$ be an anticanonical surface and let $L$ be a line bundle satisfying property $N_{p}$ but not property $N_{p+1}$.

a) If $X=\mathbf{P}^{2}$ and $m>\frac{p}{K_{X}^{2}}$;

b) if $X=\mathbf{F}_{e}$ and $m>\frac{p-e-1}{K_{X}^{2}}$;

c) if $1 \leq K_{X}^{2} \leq 7$, and $m>\frac{p+3}{K_{X}^{2}}-1$;

d) if $1 \leq K_{X}^{2} \leq 7, L$ is not a multiple of $-K_{X}$ and $m>\frac{p+1}{K_{X}^{2}}-1$;

e) if $K_{X}^{2}<0$, and $m<\frac{p+2}{K_{X}^{2}}$,

then $m K_{X}+L$ is not ample.

Proof. We outline the proof of c), d), e); a) and b) are similar. Let $1 \leq K_{X}^{2} \leq 7$ and assume $m K_{X}+L$ is ample. Then $-K_{X} \cdot\left(m K_{X}+L\right) \geq K_{X}^{2}$ by Proposition 1.10. This implies $-K_{X} \cdot L \geq(m+1) K_{X}^{2}$. Now if $L$ satisfies $N_{p}$ but not $N_{p+1},-K_{X} \cdot L=p+3$. Then $(m+1) K_{X}^{2} \leq p+3$, and $m \leq \frac{p+3}{K_{X}^{2}}-1$. If $L$ is not a multiple of $-K_{X}$, then $-K_{X} \cdot\left(m K_{X}+L\right) \geq K_{X}^{2}+2$ and we argue similarly.

Let now $K_{X}^{2}<0$ and assume again that $m K_{X}+L$ is ample. Then $-K_{X} \cdot\left(m K_{X}+L\right) \geq 1$. This implies $-K_{X} \cdot L \geq m K_{X}^{2}+1$. Again, if $L$ satisfies $N_{p}$ but not $N_{p+1},-K_{X} \cdot L=p+3$. Then $m K_{X}^{2}+1 \leq p+3$, and $m \geq \frac{p+2}{K_{X}^{2}}$. 
Remark 1.30. The bounds for $m$ in the previous theorem are sharp. That is clear for the bound in a). For the bound in b) take $L=C_{0}+(e+1) f$. For the bound in c) take $L=n\left(-K_{X}\right)$. For the bound in d) take $L=A$, where $A$ is as in Example 1.16.

\section{FANO $n$-FOLDS OF INDEX GREATER THAN OR EQUAL TO $n-1$}

In this section and in the next we prove results on syzygies of certain Fano $n$-folds. The first attempt one would try to make to tackle this problem is to imitate the arguments we carried on in Section 1. Given a Fano $n$-fold $X$ and a very ample line bundle $L, H^{1}(r L)=0$ for all $r \geq 0$. Then one could try to obtain information about the free resolution of the image of $X$ from whatever information is available on the free resolution of its general hyperplane section $X^{\prime}$, which is now of dimension $n-1$. If no information is readily available on the resolution of $X^{\prime}$, one would iterate the argument, and taking successive hyperplane sections one could read the Betti numbers of the resolution of $X$ from the resolution of a surface or even, of a curve. This worked very well in the case of rational surfaces because we ended with a curve $C$ and a line bundle $L_{C}$ on $C$ of relatively high degree, and because of this high degree, we knew relevant information about the resolution of the embedding of $C$ by $\left|L_{C}\right|$, thanks to the results of Green and Lazarsfeld. However when the dimension is higher we lose control of the hyperplane sections of $X$, or rather, there is much less information available on the syzygies of the hyperplane sections.

We will look at one example to explain what we mean. Let $X$ be $\mathbf{P}^{n}$ and let $L=\mathcal{O}_{\mathbf{P}^{n}}(d)$. If we consider the intersection of $n-2$ general divisors of $|L|$ we end with a surface in $\mathbf{P}^{n}$ which is a $(d, \ldots, d)$ complete intersection. The only surfaces among those which are rational surfaces (in fact, anticanonical) other than linear $\mathbf{P}^{2}$ are the quadric and the cubic hypersurface in $\mathbf{P}^{3}$ and a $(2,2)$ complete intersection in $\mathbf{P}^{4}$. This means that, using essentially the same ideas as in Section 1 , one is able to give a result as precise as Theorem 1.3 regarding the property $N_{p}$ for $X=\mathbf{P}^{3}$ and $L=\mathcal{O}_{\mathbf{P}^{2}}(2), \mathcal{O}_{\mathbf{P}^{2}}(3)$ and for $X=\mathbf{P}^{4}$ and $L=\mathcal{O}_{\mathbf{P}^{2}}(2)$. Precisely one has that $\mathcal{O}_{\mathbf{P}^{3}}(2)$ satisfies property $N_{5}$ but not $N_{6}, \mathcal{O}_{\mathbf{P}^{3}}(3)$ satisfies property $N_{6}$ but not $N_{7}$ and $\mathcal{O}_{\mathbf{P}^{4}}(2)$ satisfies property $N_{5}$ but not $N_{6}$. These particular cases were known (cf. [JPW] and [OP]). However for other pairs $(X, L)$ the game turns out to be much more complicated. For instance, if $(X, L)=\left(\mathbf{P}^{3}, \mathcal{O}_{\mathbf{P}^{3}}(4)\right)$ or $\left(\mathbf{P}^{5}, \mathcal{O}_{\mathbf{P}^{5}}(2)\right)$ by the above process we arrive at a K3 surface. Then knowing the syzygies of a K3 surfaces is equivalent to knowing the syzygies of its hyperplane section, a canonical curve. On this much less information is known and moreover, this information would depend (at least conjecturally) on the Clifford index of the hyperplane section. Finally all the other complete intersection surfaces of type $(d, \ldots, d)$ are surfaces of general type. Then $L_{C}$ will be a line bundle on the hyperplane section $C$ of degree strictly less than $2 g(C)-2$, and for those line bundles our knowledge is even more incomplete than for the canonical line bundle.

Because of all the above we need to carry out different arguments in this and in the following section, but before that we will state Theorem 2.1, which is based upon the work done in Section 1. Theorem 2.1 gives a necessary and sufficient condition for the line bundle $H$ giving the index of the Fano $n$-fold of index $n-1$ to satisfy property $N_{p}$. Since a Fano $n$-fold $(X, H)$ of index $n+1$ or $n$ is $\left(\mathbf{P}^{n}, \mathcal{O}_{\mathbf{P}^{n}}(1)\right)$ or a quadric hypersurface, we do not consider Fano $n$-folds of these indices in the theorem:

Theorem 2.1. Let $X$ be a Fano $n$-fold. Assume there exists an ample and base-point-free line bundle $H$ such that $K_{X}^{*}=(n-1) H$ (e.g., if $X$ is a Fano $n$-fold of index $\left.n-1\right)$. Assume furthermore that $H^{n} \geq p+3$. Then $H^{1}\left(M_{r H} \otimes l H\right)=0$ for all $r, l \geq 1$ and $H$ satisfies property $N_{p}$.

Proof. The result is proven by induction on the dimension $n$ of $X$, starting the induction in dimension 
2. If $n=2$, the results follows from Theorem 1.3. Indeed, the only thing to be checked is that $-K_{X} \cdot H \geq p+3, p \geq 0$. Then $-K_{X}=H$, so we have the required inequality by hypothesis.

Let us assume the result to be true for all dimensions from 2 to $n-1$, and we will prove it for $X$ of dimension $n$. We first see that $H$ satisfies property $N_{0}$. It suffices to prove that

$$
H^{0}(r H) \otimes H^{0}(H) \stackrel{\alpha}{\rightarrow} H^{0}((r+1) H)
$$

surjects for all $r \geq 1$. Let $Y$ be a smooth irreducible member of $|H|$. By Kodaira Vanishing Theorem, $H^{1}(l H)=0$ for all $l \geq 0$, hence by Observation 1.1, it suffices to see that

$$
0 \longrightarrow H^{0}\left(r H_{Y}\right) \otimes H^{0}\left(H_{Y}\right) \stackrel{\beta}{\longrightarrow} H^{0}\left((r+1) H_{Y}\right) \longrightarrow 0
$$

surjects for all $r \geq 1$.

The variety $Y$ is a Fano $(n-1)$-fold, $K_{Y}=\left(K_{X}+H\right)_{Y}=-(n-2) H_{Y}$ and $H_{Y}^{n-1}=H^{n} \geq 3$. Therefore $\beta$ surjects by induction.

Therefore we have just proven that $H$ satisfies property $N_{0}$. To see it does satisfies property $N_{p}$, we argue as in Theorem 1.3. Since $H^{1}(l H)=0$ for all $l \geq 0, H$ satisfies the same property $N_{p}$ as $H_{Y}$. Then by induction, since $H_{Y}^{n-1}=H^{n} \geq p+3, H$ satisfies property $N_{p}$.

Remark 2.2. Theorem 2.1 is in fact a characterization of the property $N_{p}$ satisfied by $H$. This follows because the same is true for the successive hyperplane section, which is a rational surface as we explained in the proof of Theorem 2.1.

Now we want to show a result about the syzygies associated to the multiples of the line bundle $H$. As pointed out at the beginning of this section we need to use different ideas than those used in Section 1. Any result on the graded Betti numbers of the resolution of a variety can be realized in terms of Koszul cohomology. This was shown by M. Green. For a base-point-free line bundle $L$ we define the vector bundle $M_{L}$ as

$$
0 \longrightarrow M_{L} \longrightarrow H^{0}(L) \otimes \mathcal{O} \longrightarrow L \longrightarrow 0 \text {. }
$$

Then regarding property $N_{p}$ Green showed the following criterion:

Theorem 2.3 ([EL], Section 1.). Let $L$ be an ample, globally generated line bundle on a variety $X$. If the group $H^{1}\left(\bigwedge^{p^{\prime}+1} M_{L} \otimes s L\right)$ vanishes for all $0 \leq p^{\prime} \leq p$ and all $s \geq 1$, then $L$ satisfies the property $N_{p}$. If in addition $H^{1}(r L)=0$, for all $r \geq 1$, then the above is a necessary and sufficient condition for $L$ to satisfy property $N_{p}$.

According to Theorem 2.3 the results (see for instance Theorem 1.3 ) obtained in Section 1 for the syzygies of a rational surface $X$ embedded by $L$ are equivalent to the vanishing of $H^{1}\left(\wedge^{p^{\prime}+1} M_{L} \otimes s L\right)$ for all $0 \leq p^{\prime} \leq p$ and $s \geq 1$. To carry out the arguments for Fano $n$-folds, we would need however to have the vanishing of $H^{1}\left(M_{L}^{\otimes p+1} \otimes s L\right)$, which does not follow in general from the vanishing of $H^{1}\left(\wedge^{p+1} M_{L} \otimes s L\right)$. That is the reason why we prove Theorem 2.6. Before that, we need to state two auxiliary lemmas:

Observation 2.4. Let $E$ and $L_{1}, \ldots, L_{r}$ be coherent sheaves on a variety $X$. Consider the map $H^{0}(E) \otimes H^{0}\left(L_{1}+\cdots+L_{r}\right) \stackrel{\psi}{\longrightarrow} H^{0}\left(E \otimes L_{1}+\cdots+L_{r}\right)$ and the maps

$$
\begin{gathered}
H^{0}(E) \otimes H^{0}\left(L_{1}\right) \stackrel{\alpha_{1}}{\longrightarrow} H^{0}\left(E \otimes L_{1}\right), \\
H^{0}\left(E \otimes L_{1}\right) \otimes H^{0}\left(L_{2}\right) \stackrel{\alpha_{2}}{\longrightarrow} H^{0}\left(E \otimes L_{1}+L_{2}\right), \\
\cdots, \\
H^{0}\left(E \otimes L_{1}+\cdots+L_{r-1}\right) \otimes H^{0}\left(L_{r}\right) \stackrel{\alpha_{r}}{\longrightarrow} H^{0}\left(E \otimes L_{1}+\cdots+L_{r}\right) .
\end{gathered}
$$


If $\alpha_{1}, \ldots, \alpha_{r}$ are surjective then $\psi$ is also surjective.

Lemma 2.5 ([GP4], Lemma 2.9). Let $X$ be a projective variety, let $q$ be a nonnegative integer and let $F_{i}$ be a base-point-free line bundle on $X$ for all $1 \leq i \leq q$. Let $Q$ be an effective line bundle on $X$ and let $\mathfrak{q}$ be a reduced and irreducible member of $|Q|$. Let $R$ be a line bundle and $G$ a sheaf on $X$ such that

1. $H^{1}\left(F_{i} \otimes Q^{*}\right)=0$

2. $H^{0}\left(M_{\left(F_{i_{1}} \otimes \mathcal{O}_{\mathfrak{q}}\right)} \otimes \cdots \otimes M_{\left(F_{i^{\prime}} \otimes \mathcal{O}_{\mathfrak{q}}\right)} \otimes R \otimes \mathcal{O}_{\mathfrak{q}}\right) \otimes H^{0}(G) \rightarrow$

$\rightarrow H^{0}\left(M_{\left(F_{i_{1}} \otimes \mathcal{O}_{\mathfrak{q}}\right)} \otimes \cdots \otimes M_{\left(F_{i_{q^{\prime}}} \otimes \mathcal{O}_{\mathfrak{q}}\right)} \otimes R \otimes G \otimes \mathcal{O}_{\mathfrak{q}}\right)$ surjects for all $0 \leq q^{\prime} \leq q$.

Then, for all $0 \leq q^{\prime \prime} \leq q$ and any subset $\left\{j_{k}\right\} \subseteq\{i\}$ with $\#\left\{j_{k}\right\}=q^{\prime \prime}$ and for all $0 \leq k^{\prime} \leq q^{\prime \prime}$,

$$
\begin{gathered}
H^{0}\left(M_{F_{j_{1}}} \otimes \cdots \otimes M_{F_{j_{k^{\prime}}}} \otimes M_{\left(F_{j_{k^{\prime}+1}} \otimes \mathcal{O}_{\mathfrak{q}}\right)} \otimes \cdots \otimes M_{\left(F_{j_{q^{\prime \prime}}} \otimes \mathcal{O}_{\mathfrak{q}}\right)} \otimes R \otimes \mathcal{O}_{\mathfrak{q}}\right) \otimes H^{0}(G) \rightarrow \\
H^{0}\left(M_{F_{j_{1}}} \otimes \cdots \otimes M_{F_{j_{k^{\prime}}}} \otimes M_{\left(F_{j_{k^{\prime}+1}} \otimes \mathcal{O}_{\mathfrak{q}}\right)} \otimes \cdots \otimes M_{\left(F_{j_{q^{\prime \prime}}} \otimes \mathcal{O}_{\mathfrak{q}}\right)} \otimes G \otimes R \otimes \mathcal{O}_{\mathfrak{q}}\right)
\end{gathered}
$$

surjects.

Now we are ready to prove

Theorem 2.6. Let $X$ be a rational surface. Let $B$ be an ample and base-point-free line bundle such that $-K_{X} \cdot B \geq 4$ or $(X, B)=\left(\mathbf{P}^{2}, \mathcal{O}_{\mathbf{P}^{2}}(1)\right)$. Then $H^{1}\left(M_{r B}^{\otimes p+1} \otimes l B\right)=0$ for all $r \geq 1, l \geq p$ and $p \geq 1$. In particular $l B$ satisfies property $N_{p}$ for all $l \geq p$.

Proof. We first do the case $-K_{X} \cdot B \geq 4$. The proof goes by induction on $p$. We start proving the case $p=1$. We want to show that $H^{1}\left(M_{r B}^{\otimes 2} \otimes l B\right)=0$ for all $r \geq 1$ and all $l \geq 1$. We tensor $\left(^{*}\right)$ associated to $r B$ by $M_{r B} \otimes l B$ and take global sections. As a piece of the long exact sequence of cohomology we obtain:

$$
\begin{aligned}
& H^{0}\left(M_{r B} \otimes l B\right) \otimes H^{0}(r B) \stackrel{\alpha}{\longrightarrow} H^{0}\left(M_{r B} \otimes(r+l) B\right) \\
& \longrightarrow H^{1}\left(M_{r B}^{\otimes 2} \otimes l B\right) \longrightarrow H^{1}\left(M_{r B} \otimes l B\right) \otimes H^{0}(r B) .
\end{aligned}
$$

By Theorem 1.3, the last term of the above sequence is 0 . Then the vanishing of $H^{1}\left(M_{r B}^{\otimes 2} \otimes l B\right)$ is equivalent to the surjectivity of $\alpha$. To have the surjectivity of $\alpha$, by Observation 2.4 it suffices to see the surjectivity of

$$
H^{0}\left(M_{r B} \otimes l B\right) \otimes H^{0}(B) \stackrel{\beta}{\rightarrow} H^{0}\left(M_{r B} \otimes(l+1) B\right) .
$$

Since $B$ is base-point-free and ample, we can choose $C$ smooth and irreducible in $|B|$. Then to see the surjectivity of $\beta$, by Observation 1.1 and Lemma 2.5, it suffices to see the surjectivity of

$$
H^{0}\left(M_{r B_{C}} \otimes l B_{C}\right) \otimes H^{0}\left(B_{C}\right) \stackrel{\gamma}{\rightarrow} H^{0}\left(M_{r B_{C}} \otimes(l+1) B_{C}\right) .
$$

To obtain the surjectivity of $\gamma$ we show that the cokernel of $\gamma$ vanishes. The cokernel of $\gamma$ is $H^{1}\left(M_{r B_{C}}^{\otimes 2} \otimes l B_{C}\right)$. Since $-K_{X} \cdot B \geq 4, \operatorname{deg} B_{C} \geq 2 g(C)+2$, then by [B], Theorem $1.12, M_{r B_{C}}$ is semistable, and by [Mi], Corollary 3.7 so is $M_{r B_{C}}^{\otimes 2} \otimes l B_{C}$. A simple calculation shows that $\mu\left(M_{r B_{C}}^{\otimes 2} \otimes\right.$ $\left.l B_{C}\right)>2 g(C)-2$ and we get the desired vanishing.

Now we assume the result to be true for $1, \ldots, p-1$ and we will prove it for $p$, i.e., we want to see that $H^{1}\left(M_{r B}^{\otimes p+1} \otimes l B\right)=0$. We go over the steps given to prove the case $p=1$. Since by induction $H^{1}\left(M_{r B}^{\otimes p} \otimes l B\right)=0$, the vanishing we seek is equivalent to the surjectivity of 


$$
H^{0}\left(M_{r B}^{\otimes p} \otimes l B\right) \otimes H^{0}(r B) \stackrel{\alpha}{\longrightarrow} H^{0}\left(M_{r B}^{\otimes p} \otimes(r+l) B\right) .
$$

By Observation 2.4 it suffices to see that

$$
H^{0}\left(M_{r B}^{\otimes p} \otimes l B\right) \otimes H^{0}(B) \stackrel{\beta}{\rightarrow} H^{0}\left(M_{r B}^{\otimes p} \otimes(l+1) B\right)
$$

surjects. Now choosing an irreducible and smooth curve $C$ in $|B|$ and using Observation 1.1 and Lemma 2.5,

we see that it is enough to show that

$$
H^{0}\left(M_{r B_{C}}^{\otimes p} \otimes l B_{C}\right) \otimes H^{0}\left(B_{C}\right) \stackrel{\gamma}{\rightarrow} H^{0}\left(M_{r B_{C}}^{\otimes p} \otimes(l+1) B_{C}\right)
$$

surjects.

Finally $\gamma$ surjects if $H^{1}\left(M_{r B_{C}}^{\otimes p+1} \otimes l B_{C}\right)=0$. By [B], Theorem 1.12 and [Mi], Corollary 3.7, the bundle $M_{r B_{C}}^{\otimes p+1} \otimes l B_{C}$ is semistable. On the other hand its slope $\mu$ is

$$
\frac{-(p+1) r B^{2}}{r B^{2}-g(C)}+l B^{2}
$$

so we will conclude our argument if we see that $\mu>2 g(C)-2$. This follows from $l \geq p \geq 2,-K_{X} \cdot B \geq$ 4.

As in the case $p=1$, one can alternatively deduce the surjectivity of $\gamma$ from [B], Proposition 2.2.

To finish the proof we take care of the case $(X, B)=\left(\mathbf{P}^{2}, \mathcal{O}_{\mathbf{P}^{2}}(1)\right.$. The proof goes along the same lines as before. We want the vanishing of $H^{1}\left(M_{r B}^{\otimes p+1} \otimes l B\right)$ for all $r \geq 1$ and all $l \geq p$. This follows from the vanishing of $H^{1}\left(M_{\mathcal{O}_{\mathbf{P}^{1}(r)}}^{\otimes p+1} \otimes \mathcal{O}_{\mathbf{P}^{1}}(l)\right)$, which can be easily checked as $M_{\mathcal{O}_{\mathbf{P}^{1}(r)}}$ is direct sum of copies of $\mathcal{O}_{\mathbf{P}^{1}}(-1)$.

We use Theorem 2.6 to obtain the following results on Koszul cohomology and syzygies for multiples of $H$.

Theorem 2.7. Let $X$ be a Fano $n$-fold. Assume there exists an ample and base-point-free line bundle $H$ such that $-K_{X}=m H$, with $m \geq n-1$ (e.g., if $X$ is a Fano $n$-fold of index $m \geq n-1$ ). Assume furthermore that $H^{n} \geq 4$ if $m=n-1$. Then $H^{1}\left(M_{r H}^{\otimes p+1} \otimes l H\right)=0$ for all $r \geq 1, l \geq p$ and $p \geq 1$.

Proof. To prove $H^{1}\left(M_{r H}^{\otimes p+1} \otimes l H\right)=0$ we argue by induction on the dimension of $X$, the cornerstone being now Theorem 2.6, and by induction on $p$.

Let first $p=1$. We want to obtain the vanishing of $H^{1}\left(M_{r H}^{\otimes 2} \otimes l H\right)$ and, as announced, we do it by induction on the dimension $n$. If $n=2$ the result is a particular case of Theorem 2.6. In fact, $-K_{X}=m H$ with $m \geq 1$. If $m=1,-K_{X} \cdot H \geq 4$ follows directly by hypothesis. If $m \geq 2$, $-K_{X} \cdot H \geq 2$, since $H$ is ample, and $-K_{X} \cdot H \geq 4$ unless $(X, H)=\left(\mathbf{P}^{2}, \mathcal{O}_{\mathbf{P}^{2}}(1)\right)$.

Now we assume the result to be true for dimensions 2 to $n-1$ and we will prove it for dimension $n$. From $(*)$ we obtain

$$
\begin{aligned}
& H^{0}\left(M_{r H} \otimes l H\right) \otimes H^{0}(r H) \stackrel{\alpha}{\longrightarrow} H^{0}\left(M_{r H} \otimes(r+l) H\right) \\
& \longrightarrow H^{1}\left(M_{r H}^{\otimes 2} \otimes l H\right) \longrightarrow H^{1}\left(M_{r H} \otimes l H\right) \otimes H^{0}(r H) .
\end{aligned}
$$


By the vanishing of the first cohomology of the multiples of $H$, Theorem 2.1 implies the vanishing of the last term of the above sequence. Then the vanishing we seek is equivalent to the surjectivity of $\alpha$. By Observation 2.4 we see that it will suffice to prove that

$$
H^{0}\left(M_{r H} \otimes l H\right) \otimes H^{0}(H) \stackrel{\beta}{\rightarrow} H^{0}\left(M_{r H} \otimes(l+1) H\right)
$$

surjects for all $r, l \geq 1$. The conditions needed to apply Observation 1.1 and Lemma 2.5 follow from Theorem 2.1 and from the fact that $H^{1}\left(M_{r H}\right)=0$, for $H^{1}\left(\mathcal{O}_{X}\right)=0$. Then it suffices to see

$$
H^{0}\left(M_{r H_{Y}} \otimes l H_{Y}\right) \otimes H^{0}\left(H_{Y}\right) \stackrel{\gamma}{\rightarrow} H^{0}\left(M_{r H_{Y}} \otimes(l+1) H_{Y}\right)
$$

surjects, which follows because the result is true for $p=1$ and $Y$ of dimension $n-1$, by induction hypothesis.

Now we assume the result to be true for $1, \ldots, p-1$ and we will prove it for $p$. We argue again by induction on the dimension. In dimension 2 the result follows from Theorem 2.6 as in the case $p=1$. We therefore assume the result to be true for $p$ and dimensions 2 to $n-1$ and we will prove it for $p$ and dimension $n$, i.e., we will prove $H^{1}\left(M_{r H}^{\otimes p+1} \otimes l H\right)=0$ for all $r \geq 1, l \geq p$. From $\left(^{*}\right)$ we obtain

$$
\begin{gathered}
H^{0}\left(M_{r H}^{\otimes p} \otimes l H\right) \otimes H^{0}(r H) \stackrel{\alpha}{\longrightarrow} H^{0}\left(M_{r H}^{\otimes p} \otimes(r+l) H\right) \\
\longrightarrow H^{1}\left(M_{r H}^{\otimes p+1} \otimes l H\right) \longrightarrow H^{1}\left(M_{r H}^{\otimes p} \otimes l H\right) \otimes H^{0}(r H) .
\end{gathered}
$$

By induction hypothesis on $p$, we have the vanishing of the last term of the above sequence. Therefore the vanishing we seek is equivalent to the surjectivity of $\alpha$. By Observation 2.4 we see that it will suffice to prove that

$$
H^{0}\left(M_{r H}^{\otimes p} \otimes l H\right) \otimes H^{0}(H) \stackrel{\beta}{\rightarrow} H^{0}\left(M_{r H}^{\otimes p} \otimes(l+1) H\right)
$$

surjects for all $r \geq 1, l \geq p$. The conditions needed to apply Observation 1.1 and Lemma 2.5

follow from induction hypothesis on $p$. Then it suffices to see

$$
H^{0}\left(M_{r H_{Y}}^{\otimes p} \otimes l H_{Y}\right) \otimes H^{0}\left(H_{Y}\right) \stackrel{\gamma}{\rightarrow} H^{0}\left(M_{r H_{Y}}^{\otimes p} \otimes(l+1) H_{Y}\right)
$$

surjects, which follows because the result is true for $p$ and $Y$ of dimension $n-1$, by induction hypothesis on $n$.

Corollary 2.8. Let $X$ be a Fano $n$-fold. Assume there exists an ample and base-point-free line bundle $H$ such that $-K_{X}=m H$, with $m \geq n-1$ (e.g., if $X$ is a Fano $n$-fold of index $m \geq 1$ ). Assume furthermore that $H^{n} \geq 4$ if $m=n-1$. Then $l H$ satisfies property $N_{p}$ for all $l \geq p$.

Proof.

Since we work in characteristic 0 , then

$$
H^{1}\left(\bigwedge^{\otimes i} M_{l H} \otimes s l H\right)=0, \text { for all } l \geq p, s \geq 1,1 \leq i \leq p+1
$$

Therefore, according to [GL], $l H$ satisfies property $N_{p}$. 


\section{FANO $n$-FOLDS OF INDEX $n-3$.}

In this last section we deal with Fano $n$-folds of index $n-3, n \geq 4$. We use the same ideas as in the second part of Section 2. We start by studying under what conditions $m H$ is very ample and satisfies property $N_{0}$ :

Theorem 3.1. Let $X$ be a Fano $n$-fold such that $-K_{X}=m H, H$ is ample and base-point-free, and $m=n-3 \geq 1$ (for instance, if $X$ is a Fano $n$-fold of index $m=n-3$ ). Let $L=k H$.

(1) If $k \geq 4$, then $L$ satisfies property $N_{0}$.

(2) Let $k=3$; L satisfies property $N_{0}$ if and only if the morphism induced by $|H|$ does not map $X 2: 1$ onto $\mathbf{P}^{n}$.

(3) Let $k=2$; if $|H|$ does not map $X$ onto a variety of minimal degree other than $\mathbf{P}^{n}$ nor maps $X 2: 1$ onto $\mathbf{P}^{n}$, then $L$ satisfies property $N_{0}$.

Proof. We will prove the result by induction on $n$. We start at $n=4$. We have to deal with several cases.

Case 1: $k \geq 4$. We want to prove that $L=k H$ satisfies property $N_{0}$, or equivalently, that

$$
H^{0}(s k H) \otimes H^{0}(k H) \longrightarrow H^{0}((s+1) k H)
$$

surjects for all $s \geq 1$. This follows from a more general result, namely,

$$
H^{0}(k H) \otimes H^{0}(H) \stackrel{\alpha}{\longrightarrow} H^{0}((k+1) H)
$$

surjects for all $k \geq 4$. Indeed, the surjectivity of $\alpha$ follows from [Mu], p. 41, Theorem 2 , since by Kodaira Vanishing Theorem, $H$ is 3-regular.

Case 2: $k=3$. We distinguish two subcases.

Case 2.1: First let us assume that $|H|$ does not map $X$ onto $\mathbf{P}^{4}$. We will show

$$
H^{0}(k H) \otimes H^{0}(H) \stackrel{\alpha}{\longrightarrow} H^{0}((k+1) H)
$$

for all $k \geq 3$. If $k \geq 4$, we have already seen that $\alpha$ surjects. To show the surjectivity for $k=3$, let $Y$ be a smooth irreducible member of $|H|$. By Observation 1.1, since $H^{1}(l H)=0$, for all $l \geq 0$ by Kodaira Vanishing Theorem, it will suffice to check that

$$
H^{0}\left(3 H_{Y}\right) \otimes H^{0}\left(H_{Y}\right) \stackrel{\beta}{\longrightarrow} H^{0}\left(4 H_{Y}\right)
$$

surjects. By adjunction, $\left(Y, H_{Y}\right)$ is a polarized Calabi-Yau threefold, and, since $H^{1}\left(\mathcal{O}_{X}\right)=0,\left|H_{Y}\right|$ does not map $Y$ onto $\mathbf{P}^{3}$. Then $\beta$ surjects (cf. [GP3], proof of Theorem 1.4, case 1).

Case 2.2: Now assume $|H|$ does map $X$ onto $\mathbf{P}^{4}$. We assume first that the map induced by $|H|$ is not $2: 1$ and we will prove that

$$
H^{0}(3 l H) \otimes H^{0}(3 H) \longrightarrow H^{0}(3(l+1) H)
$$

surjects for all $l \geq 1$. For that, by Observation 2.4 it is enough that

$$
\begin{gathered}
H^{0}(r H) \otimes H^{0}(H) \stackrel{\alpha}{\rightarrow} H^{0}((r+1) H) \text { for all } r \geq 5 \\
H^{0}(3 H) \otimes H^{0}(2 H) \stackrel{\gamma}{\rightarrow} H^{0}(5 H)
\end{gathered}
$$


surject. The map $\alpha$ was seen to be surjective in Case 1. For the surjectivity of $\gamma$ we choose smooth irreducible $Y$ in $|H|$ and we write the following commutative diagram,

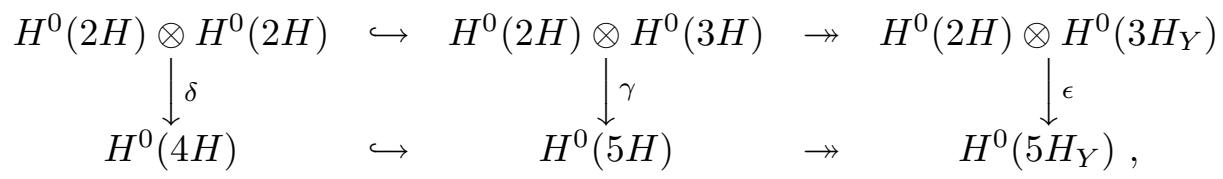

obtained from the sequence

$$
0 \longrightarrow H^{*} \longrightarrow \mathcal{O}_{X} \longrightarrow \mathcal{O}_{Y} \longrightarrow 0
$$

having in account that $H^{1}(r H)=0$ for all $r \geq 0$. To see the surjectivity of $\delta$ we construct another diagram arising from (3.1.1):

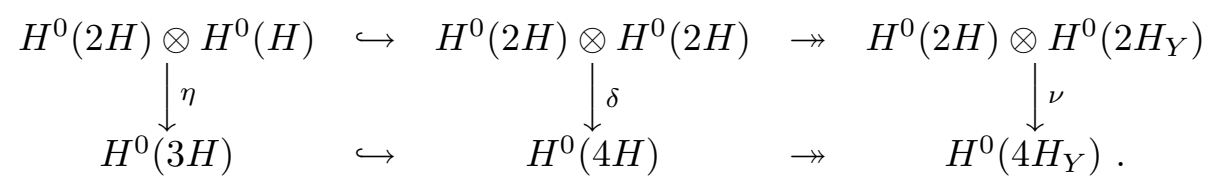

Thus we need to see that $\eta, \epsilon$ and $\nu$ are surjective. By Observation 1.1, for the surjectivity of $\eta$ it suffices to see the surjectivity of

$$
H^{0}\left(2 H_{Y}\right) \otimes H^{0}\left(H_{Y}\right) \stackrel{\mu}{\rightarrow} H^{0}\left(3 H_{Y}\right)
$$

and for the surjectivity of $\epsilon$ and $\nu$, since $H^{1}(H)=0$, it suffices to see that

$$
\begin{aligned}
& H^{0}\left(3 H_{Y}\right) \otimes H^{0}\left(2 H_{Y}\right) \stackrel{\pi}{\rightarrow} H^{0}\left(5 H_{Y}\right) \\
& H^{0}\left(2 H_{Y}\right) \otimes H^{0}\left(2 H_{Y}\right) \stackrel{\rho}{\rightarrow} H^{0}\left(4 H_{Y}\right)
\end{aligned}
$$

both surject. $\left(Y, H_{Y}\right)$ is a polarized Calabi-Yau threefold such that the morphism induced by $\left|H_{Y}\right|$ maps $Y$ onto to $\mathbf{P}^{3}$ but is not $2: 1$. Then the surjectivity of $\pi$ and $\rho$ is explicitly claimed (and proved) in the proof of Theorem 1.4, [GP3] and the surjectivity of $\mu$ is also proved there, although not explicitly said.

To end Case 2.2 assume $|H|$ maps $X 2: 1$ onto $\mathbf{P}^{4}$. If $Y$ is a smooth and irreducible member of $|H|$, then $\left|H_{Y}\right|$ maps $Y 2: 1$ onto $\mathbf{P}^{3}$, so according to [GP3], Theorem $1.4, H_{Y}$ is not very ample, nor is $H$.

Case 3: $k=2$. We want to see that if $H$ is ample, base-point-free and $|H|$ does not map $X$ onto a variety of minimal degree (different from $\mathbf{P}^{4}$ ), nor does it map $X 2: 1$ onto $\mathbf{P}^{4}$, then

$$
H^{0}(2 l H) \otimes H^{0}(2 H) \longrightarrow H^{0}((2 l+2) H)
$$

surjects for all $l \geq 1$.

Case 3.1: Assume $h^{0}(H) \geq 6$. By Observation 2.4, it suffices to prove that

$$
H^{0}(r H) \otimes H^{0}(H) \stackrel{\alpha}{\longrightarrow} H^{0}((r+1) H)
$$

surjects for all $r \geq 2$. If $r \geq 3$, we have seen that $\alpha$ surjects while proving Case 1 and Case 2.1. To see that

$$
H^{0}(2 H) \otimes H^{0}(H) \stackrel{\alpha}{\longrightarrow} H^{0}(3 H)
$$


surjects let $Y$ be as before a smooth and irreducible member of $|H|$. By Observation 1.1 it suffices to see that

$$
H^{0}\left(2 H_{Y}\right) \otimes H^{0}\left(H_{Y}\right) \stackrel{\beta}{\rightarrow} H^{0}\left(3 H_{Y}\right)
$$

surjects. Now $\left(Y, H_{Y}\right)$ is a polarized Calabi-Yau satisfying the hypothesis of Theorem 1.7.1, [GP3]. Then the map $\beta$ surjects, as seen in the proof of Theorem 1.7, [GP3].

Case 3.2: Assume now that $h^{0}(H)=5$ and that the map induced by $|H|$ from $X$ onto $\mathbf{P}^{4}$ is not $2: 1$. By Observation 2.4 it suffices to see that

$$
\begin{gathered}
H^{0}(r H) \otimes H^{0}(H) \longrightarrow H^{0}((r+1) H) \text { for all } r \geq 4 \text { and } \\
H^{0}(2 H) \otimes H^{0}(2 H) \longrightarrow H^{0}(4 H)
\end{gathered}
$$

surject, and this has been proved in Case 1 and Case 2.2 .

To prove the result for a Fano variety of arbitrary dimension $n$ we will argue by induction. Let $Y$ be smooth and irreducible member of $|H|$. If $-K_{X}=(n-3) H$, then $-K_{Y}=(n-2) H_{Y}$ and $h^{0}\left(H_{Y}\right)=h^{0}(H)-1$. Moreover, if the image of $X$ by the morphism induced by $|H|$ is a variety of minimal degree, so is the image of $Y$ by the morphism induced by $\left|H_{Y}\right|$, and the degree of both morphisms is the same. Going over the arguments in the case $n=4$, we see that the key point was to show the surjectivity of the maps

$$
\begin{gathered}
H^{0}(r H) \otimes H^{0}(H) \stackrel{\alpha}{\rightarrow} H^{0}((r+1) H) \text { for all } k \geq 4, \\
H^{0}(3 H) \otimes H^{0}(H) \stackrel{\beta}{\rightarrow} H^{0}(4 H) \text { if } h^{0}(H) \geq n+2 \\
H^{0}(2 H) \otimes H^{0}(H) \stackrel{\gamma}{\rightarrow} H^{0}(3 H) \text { if } h^{0}(H) \geq n+2 \text { and the image of } X
\end{gathered}
$$

by the map induced by $|H|$ is not a variety of minimal degree

$H^{0}(3 H) \otimes H^{0}(2 H) \stackrel{\delta}{\rightarrow} H^{0}(5 H)$ if $h^{0}(H)=n+1$ and the map induced by $|H|$ is not $2: 1$

$H^{0}(2 H) \otimes H^{0}(2 H) \stackrel{\epsilon}{\rightarrow} H^{0}(4 H)$ if $h^{0}(H)=n+1$ and the map induced by $|H|$ is not $2: 1$

$H^{0}(2 H) \otimes H^{0}(H) \stackrel{\eta}{\rightarrow} H^{0}(3 H)$ if $h^{0}(H)=n+1$ and the map induced by $|H|$ is not $2: 1$.

Let us assume therefore the surjectivity of all the above maps for Fano varieties $(X, H)$ of dimension $4, \ldots, n-1$. Arguing as in the case $n=4$, by Observation 1.1 and because $H^{1}(r H)=0$ for all $r \geq 0$, we have that all the above maps are also surjective if the dimension of $X$ is $n$.

Then by Observation 2.4 the surjectivity of $\alpha$ implies (1), the surjectivity of $\alpha, \beta$ and $\delta$ implies the "if" part of (2), and the surjectivity of $\alpha, \beta, \gamma$ and $\epsilon$ implies (3). Finally, if the morphism induced by $|H|$ is a double cover of $\mathbf{P}^{n}$, so is the morphism induced on $Y$ by $\left|H_{Y}\right|$, when $Y$ is an irreducible and smooth member of $|H|$. Then $3 H_{Y}$ is not very ample (by induction hypothesis), nor is $3 H$.

Theorem 3.2. Let $X$ be a Fano $n$-fold of index $m=n-3$. Assume that $-K_{X}=m H$, and $H$ is ample and base-point-free. Let $L=k H$. Assume furthermore that $h^{0}(H) \geq n+2$, i.e., that $|H| d o$ not map $X$ onto $\mathbf{P}^{n}$. If $k \geq p+2$ and $p \geq 1$, then $L$ satisfies property $N_{p}$.

Proof. The proof is again by induction on the dimension. The first step is $n=4$. Given $p \geq 1$, we want to prove that

$$
H^{1}\left(M_{L}^{\otimes p+1} \otimes s L\right)=0 \text { if } L=k H, k \geq p+2, l \geq 1 .
$$


We will prove this more general fact, namely that

$$
H^{1}\left(M_{k H}^{\otimes p+1} \otimes s H\right)=0 \text { for all } k \geq p+2, s \geq p+2 .
$$

We argue now by induction on $p$. If $p=1$, we want to prove that

$$
H^{1}\left(M_{k H}^{\otimes 2} \otimes s H\right)=0 \text { for all } k \geq 3, s \geq 3 .
$$

Since $H^{1}\left(M_{k H} \otimes s H\right)=0$ by Theorem 3.1, then (3.2.3) is equivalent to the surjectivity of

$$
H^{0}\left(M_{k H} \otimes s H\right) \otimes H^{0}(k H) \longrightarrow H^{0}\left(M_{k H} \otimes(k+s) H\right) \text { for all } k \geq 3, s \geq 3,
$$

and by Observation 2.4 it suffices to see that

$$
H^{0}\left(M_{k H} \otimes H\right) \otimes H^{0}(H) \longrightarrow H^{0}\left(M_{k H} \otimes(k+1) H\right) \text { surjects for all } k \geq 3 .
$$

Choose smooth and irreducible 3-fold $Y$ in $|H|$. Because of Theorem 3.1, Observation 1.1 and Lemma 2.5 it suffices to have the surjectivity of

$$
H^{0}\left(M_{k H_{Y}} \otimes H_{Y}\right) \otimes H^{0}\left(H_{Y}\right) \longrightarrow H^{0}\left(M_{k H_{Y}} \otimes(k+1) H_{Y}\right)
$$

for all $k \geq 3$. By adjunction $Y$ is a Calabi-Yau threefold and since $H^{1}\left(\mathcal{O}_{X}\right)=0, h^{0}\left(H_{Y}\right) \geq 5$, hence from the proof of [GP3], Theorem 1.4, the above map is surjective.

We complete now the proof of the result for $n=4$. We may assume the result proved until $p-1$. Now we want to see that

$$
H^{1}\left(M_{k H}^{\otimes p+1} \otimes s H\right)=0 \text { for all } k \geq p+2, s \geq p+2 .
$$

We argue similarly to the case $p=1$. By induction on $p$ we may conclude that the sought vanishing is equivalent to the surjectivity of

$$
H^{0}\left(M_{k H}^{\otimes p} \otimes s H\right) \otimes H^{0}(k H) \longrightarrow H^{0}\left(M_{k H}^{\otimes p} \otimes(k+s) H\right) \text { for all } k \geq p+2, s \geq p+2,
$$

and by Observation 2.4 it suffices to see that

$$
H^{0}\left(M_{k H}^{\otimes p} \otimes H\right) \otimes H^{0}(H) \longrightarrow H^{0}\left(M_{k H}^{\otimes p} \otimes(k+1) H\right) \text { surjects for all } k \geq p+2 .
$$

Finally we choose a smooth and irreducible 3-fold $Y$ in $|H|$. Because of Theorem 3.1, Observation 1.1 and Lemma 2.5 it suffices to have the surjectivity of

$$
H^{0}\left(M_{k H_{Y}} \otimes H_{Y}\right) \otimes H^{0}\left(H_{Y}\right) \longrightarrow H^{0}\left(M_{k H_{Y}} \otimes(k+1) H_{Y}\right)
$$

for all $k \geq p+2$. By adjunction $Y$ is a Calabi-Yau threefold and since $H^{1}\left(\mathcal{O}_{X}\right)=0, h^{0}\left(H_{Y}\right) \geq 5$, hence according to the proof of [GP3], Theorem 1.4, the above map is surjective.

Now assume $n>4$. Recall that we want to show that

$$
H^{1}\left(M_{L}^{\otimes p+1} \otimes s L\right)=0 \text { if } L=k H, k \geq p+2, l \geq 1,
$$

and as before we will prove this more general fact, namely that

$$
H^{1}\left(M_{k H}^{\otimes p+1} \otimes s H\right)=0 \text { for all } k \geq p+2, s \geq p+2 .
$$


We argue now by induction on $p$ and $n$. If $p=1$, we want to prove that

$$
H^{1}\left(M_{k H}^{\otimes 2} \otimes s H\right)=0 \text { for all } k \geq 3, s \geq 3 .
$$

Since $H^{1}\left(M_{k H} \otimes s H\right)=0$ by Theorem 3.1, then (3.2.7) is equivalent to the surjectivity of

$$
H^{0}\left(M_{k H} \otimes s H\right) \otimes H^{0}(k H) \longrightarrow H^{0}\left(M_{k H} \otimes(k+s) H\right) \text { for all } k \geq 3, s \geq 3,
$$

and by Observation 2.4 it suffices to see that

$$
H^{0}\left(M_{k H} \otimes H\right) \otimes H^{0}(H) \stackrel{\alpha}{\rightarrow} H^{0}\left(M_{k H} \otimes(k+1) H\right) \text { surjects for all } k \geq 3 .
$$

The surjectivity of this map has been proven under the hypothesis of the theorem, when the dimension $n$ of $X$ is 4 , and we will assume it proved also if dimension of $X$ is $n-1$.

Choose smooth and irreducible $(n-1)$-fold $Y$ in $|H|$. Because of Theorem 3.1, Observation 1.1 and Lemma 2.5 it suffices to have the surjectivity of

$$
H^{0}\left(M_{k H_{Y}} \otimes H_{Y}\right) \otimes H^{0}\left(H_{Y}\right) \longrightarrow H^{0}\left(M_{k H_{Y}} \otimes(k+1) H_{Y}\right)
$$

for all $k \geq 3$. By adjunction $-K_{Y}=(n-4) H_{Y}$ and since $H^{1}\left(\mathcal{O}_{X}\right)=0, h^{0}\left(H_{Y}\right) \geq n+1$, hence by induction hypothesis, $\alpha$ surjects.

The proof of the general case follows the same steps as the proof for $n=4$. Recall that we want to proof that

$$
H^{1}\left(M_{k H}^{\otimes p+1} \otimes s H\right)=0 \text { for all } k \geq p+2, s \geq p+2 .
$$

Using now the induction hypothesis for $n-1$ one conclude the result, exactly in the same fashion as we have just done when $p=1$.

\section{REFERENCES}

[BS] M.C. Beltrametti and A.J. Sommese, The Adjunction Theory of Complex Projective Varieties, Walter de Gruyter, 1995.

[B] D. Butler, Normal generation of vector bundles over a curve, J. Differential Geometry 39 (1994) 1-34.

[EL] L. Ein and R. Lazarsfeld, Koszul cohomology and syzygies of projective varieties, Inv. Math. 111 (1993), 51-67.

[GP1] F.J. Gallego and B.P. Purnaprajna Normal presentation on elliptic ruled surfaces, J. Algebra 186, (1996), 597-625.

[GP2] _ - Higher syzygies of elliptic ruled surfaces, J. Algebra 186, (1996), 626-659.

[GP3] _ - Very ampleness and higher syzygies for Calabi-Yau threefolds, Math. Ann. 312 (1998), 133-149.

[GP4] _ Projective normality and syzygies of algebraic surfaces, J. reine angew. Math. 506 (1999), 145-180.

[GP5] _ _ Vanishing theorems and syzygies for K3 surfaces and Fano varieties, to appear in J. Pure App. Alg.

[G] M. Green, Koszul cohomology and the geometry of projective varieties, J. Differential Geometry 19 (1984), 125-171.

[GL] M. Green and R. Lazarsfeld, Some results on the syzygies of finite sets and algebraic curves, Compositio Math. 67 (1989), 301-314. 
[Hb1] B. Harbourne, Anticanonical rational surfaces, Trans. A. M. S. 349 (1997), 1191-1208.

[Hb2] _ Birational morphisms of rational surfaces, J. Algebra 190 (1997), 145-162.

[Ht] R. Hartshorne, Algebraic Geometry, Springer-Verlag 1977.

[Ho1] Y. Homma, Projective normality and the defining equations of ample invertible sheaves on elliptic ruled surfaces with $e \geq 0$, Natural Science Report, Ochanomizu Univ. 31 (1980) 61-73.

[Ho2] _ _ Projective normality and the defining equations of an elliptic ruled surface with negative invariant, Natural Science Report, Ochanomizu Univ. 33 (1982) 17-26.

[JPW] T. Josefiak, P. Pragacz and J. Weyman, Resolutions of determinantal varieties and tensor complexes associated with symmetric and antisymmetric matrix, Asterisque 87-88 (1981), 109-189.

[Mi] Y. Miyaoka, The Chern class and Kodaira dimension of a minimal variety, Algebraic Geometry -Sendai 1985, Advanced Studies in Pure Math., Vol. 10, North-Holland, Amsterdam, 449-476.

[Mu] D. Mumford Varieties defined by quadratic equations, Corso CIME in Questions on Algebraic Varieties, Rome, 1970, 30-100.

[OP] G. Ottaviani and R. Paoletti, Syzygies of Veronese embeddings, preprint.

Francisco Javier Gallego: Dpto. de Álgebra, Facultad de Matemáticas, Universidad Complutense DE MADrid, 28040 MAdrid, Spain

E-mail address: gallego@eucmos.sim.ucm.es

B.P.Purnaprajna: 405 Snow Hall, Dept. of Mathematics, University of Kansas, Lawrence, Kansas 66045-2142

E-mail address: purna@math.ukans.edu 